\title{
Profiling of chromatin accessibility identifies transcription factor binding sites across the genome of Aspergillus species
}

\author{
Lianggang Huang ${ }^{1}$, Xuejie Li ${ }^{1}$, Liangbo Dong ${ }^{1}$, Bin Wang ${ }^{1,2^{*}}$ and Li Pan ${ }^{1,2^{*}}$
}

\begin{abstract}
Background: The identification of open chromatin regions and transcription factor binding sites (TFBs) is an important step in understanding the regulation of gene expression in diverse species. ATAC-seq is a technique used for such purpose by providing high-resolution measurements of chromatin accessibility revealed through integration of Tn5 transposase. However, the existence of cell walls in filamentous fungi and associated difficulty in purifying nuclei have precluded the routine application of this technique, leading to a lack of experimentally determined and computationally inferred data on the identity of genome-wide cis-regulatory elements (CREs) and TFBs. In this study, we constructed an ATAC-seq platform suitable for filamentous fungi and generated ATAC-seq libraries of Aspergillus niger and Aspergillus oryzae grown under a variety of conditions.

Results: We applied the ATAC-seq assay for filamentous fungi to delineate the syntenic orthologue and differentially changed chromatin accessibility regions among different Aspergillus species, during different culture conditions, and among specific TF-deleted strains. The syntenic orthologues of accessible regions were responsible for the conservative functions across Aspergillus species, while regions differentially changed between culture conditions and TFs mutants drove differential gene expression programs. Importantly, we suggest criteria to determine TFBs through the analysis of unbalanced cleavage of distinct TF-bound DNA strands by Tn5 transposase. Based on this criterion, we constructed data libraries of the in vivo genomic footprint of $A$. niger under distinct conditions, and generated a database of novel transcription factor binding motifs through comparison of footprints in TF-deleted strains. Furthermore, we validated the novel TFBs in vivo through an artificial synthetic minimal promoter system.
\end{abstract}

Conclusions: We characterized the chromatin accessibility regions of filamentous fungi species, and identified a complete TFBs map by ATAC-seq, which provides valuable data for future analyses of transcriptional regulation in filamentous fungi.

Keywords: Filamentous fungi ATAC-seq, Chromatin accessibility, Footprints, Transcription factor binding sites, Aspergillus species

\footnotetext{
*Correspondence: btbinwang@scut.edu.cn; btlipan@scut.edu.cn

${ }^{1}$ School of Biology and Biological Engineering, South China University of

Technology, Guangzhou Higher Education Mega Center, Guangzhou 510006,

China

Full list of author information is available at the end of the article
}

(c) The Author(s). 2021 Open Access This article is licensed under a Creative Commons Attribution 4.0 International License, which permits use, sharing, adaptation, distribution and reproduction in any medium or format, as long as you give appropriate credit to the original author(s) and the source, provide a link to the Creative Commons licence, and indicate if changes were made. The images or other third party material in this article are included in the article's Creative Commons licence, unless indicated otherwise in a credit line to the material. If material is not included in the article's Creative Commons licence and your intended use is not permitted by statutory regulation or exceeds the permitted use, you will need to obtain permission directly from the copyright holder. To view a copy of this licence, visit http://creativecommons.org/licenses/by/4.0/ The Creative Commons Public Domain Dedication waiver (http://creativecommons.org/publicdomain/zero/1.0/) applies to the data made available in this article, unless otherwise stated in a credit line to the data. 


\section{Background}

There have been 339 species identified in the Aspergilli genus [1], and they are of broad interest because of their industrial applications, importance as pathogens for animals and crops, and relevance for basic research. Aspergillus section Nigri is the fungal genus with the most sequenced genomes, as genomes from 27 species are already publicly available [2], and genomic analyses have led to a better understanding of fungal biology and improvements in the industrial use of these organisms [1, 3 , 4]. Gene regulation is of major way by which fungi physiologically act in environmental circumstances and respond to changing conditions $[5,6]$. However, in comparison with Saccharomyces cerevisiae, the knowledge of genome-wide regulatory mechanisms in Aspergilli is lagging. Transcription factors (TFs) are key regulators of biological processes that function by binding to transcriptional regulatory regions to control the expression of target genes. The cis-regulatory elements (CREs) related to TF binding play pivotal roles in the regulation of gene expression, and each TF recognizes a collection of similar DNA sequences, known as binding site motifs [7, 8]. Therefore, the ability to identify these CREs and TF motifs throughout Aspergillus genomes is an important step in understanding the regulatory functions of $\mathrm{TF}$ binding and gene expression. Analysis of genome sequences has revealed an average of 600 putative transcription factors in each Aspergillus fungus genome [3, 9]. Nevertheless, approximately $5 \%$ of the TFs in the Aspergillus genus have only been identified and not been studied further $[3,9]$. Most genome-wide TFBs in the Aspergillus genome have not been experimentally determined or computationally inferred, and the sites remain unknown.

The gold-standard method for identifying in vivo CREs for TFs of interest is chromatin immunoprecipitation DNA sequencing (ChIP-seq) [10]. However, the scarcity of sequence-specific antibodies for most Aspergillus TFs or tagged target proteins for the more than 600 TFs in Aspergillus has prevented the widespread application of this method in Aspergillus [11]. So far, only a few ChIPseq experiments have used epitope-tagged or customized TF antibodies to study Aspergillus TFs, including CrzA [12], SrbA [13], MetR [14], and AtrR [15] of Aspergillus fumigatus; WetA of Aspergillus nidulans [16]; CRE-1 of Neurospora crassa [17]; Tri6 of Fusarium graminearum [18]; MoCRZ1 of Magnaporthe oryzae [19] and some chromatin modification such as H3K4 trimethylation in A. nidulans $[20,21]$. These findings reveal that the available filamentous fungi ChIP-seq data are not abundant and performing ChIP-seq experiments in filamentous fungi is full of challenges. Additional high-throughput sequencing methods, i.e., DNase-seq and formaldehydeassisted isolation of regulatory elements with sequencing
(FAIRE-seq) combine enzymatic digestion of isolated chromatin with sequencing, were developed to identify CREs on a genome-wide scale [22, 23]. The shortcomings of these methods are that they require millions of cells as starting material, complex and time-consuming sample preparations, and many potentially loss-prone steps, such as adaptor ligation, gel purification, and cross-link reversal [24]. For these reasons, the DNaseseq analysis that we previously carried out in Aspergillus oryzae could not be effectively replicated [23]. Therefore, the development of feasible and scalable methods is required to facilitate the identification of regulatory elements.

Assay for transposase accessible chromatin sequencing (ATAC-seq) is a recently developed technique used to identify accessible regions and DNA footprints $[25,26]$. Tn 5 transposase integrates sequencing adapters directly into DNA, eliminating the need for multiple reactions and purification steps typically required for the construction of a sequencing library. As a result, significantly lower amounts of starting nuclei are required for the investigation of CREs. ATAC-seq is now routinely being applied to systematically identify cis-regulatory regions and DNA footprints in humans [27], mice [28], zebrafish [29], Drosophila [30], Caenorhabditis elegans [31], Arabidopsis thaliana [32], S. cerevisiae [33], and so on. However, in Aspergillus species, the existence of cell walls and the purification of nuclei preparation prevented the routine application of this technique. To overcome this obstacle, we performed an ATAC-seq assay for Aspergilli by protoplast preparation under cultivation conditions. We combined ATAC-seq and RNAseq of different Aspergillus species and TF mutants to profile chromatin accessibility and identify gene regulatory elements in vivo on a genome-wide scale. Furthermore, we characterized the identified CREs in vivo by the synthetic minimal promoter driving expression of the reporter gene. Collectively, this study is an important step towards the meticulous analysis of industrial microbial transcriptional regulatory networks and provides valuable resource for future research aimed at characterizing or using gene regulatory elements.

\section{Results}

The ATAC-seq assay in the filamentous fungus

To identify chromatin accessibility and regulatory elements in filamentous fungi, we developed an ATAC-seq protocol for Aspergillus species by adding a step that released native nuclei by detergent lysis of protoplasts prior to the transposition step (Fig. 1a). A total of $5 \times$ $10^{4}$ protoplasts were subjected to the extraction of native nuclei in lysis buffer containing 0.05\% IGEPAL CA-630 (Sigma, I8896). After Tn5 transposition, PCR and electrophoresis were performed to detect the discrete 


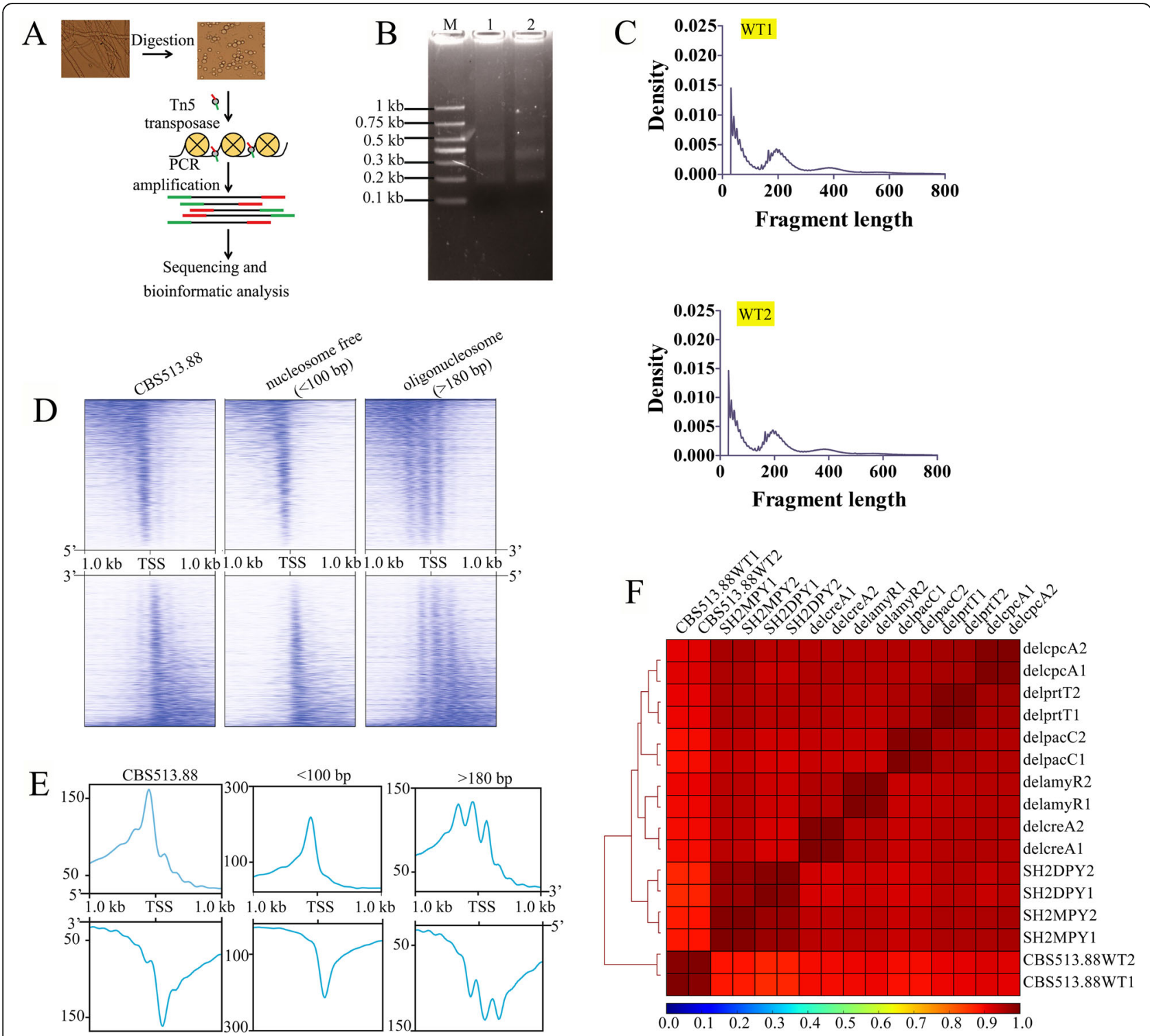

Fig. 1 Schematic illustration of the Assay for Transposase-Accessible Chromatin with high-throughput sequencing (ATAC-seq) in Aspergillus niger. a Protoplasts used for nuclei isolation and schematic procedure of ATAC-seq technique. Protoplasts were prepared under the cultivation condition by adding lywallzyme. After filtration via miracloth membrane, the concentration of extracted protoplasts reached $10^{5} / \mu \mathrm{L}$. $\mathbf{b}$ Fragment sizes for amplified ATAC-seq libraries by 15 cycles of PCR reaction. $5 \times 10^{4}$ protoplasts of A. niger CBS513.88 were used for Tn5 transposase reaction, respectively. Lane 1: replicate 1, WT1; Lane 2: replicate 2, WT2. c Insert sizes determined by high-throughput sequencing. Peaks with different fragment length indicate that the fragments contain one or more nucleosomes. $\mathbf{d}$ Heatmaps showing the distribution of accessible regions around TSSs in A. niger CBS513.88: The meaning of " < 100" is that the fragment sizes of pair-end reads were less than 100 bp of fragment size. And the meaning of " $>180$ " is that the fragment sizes of pair-end reads were more than $180 \mathrm{bp}$. Regions of $<100 \mathrm{bp}$ were assigned as nucleosome free and those of $>180 \mathrm{bp}$ were assigned as oligonucleosomal [27]. The signals are derived from two technical replicates. e Read coverage of the 1-kb region flanking TSSs in A. niger CBS513.88. $\mathbf{f}$ Heatmap clustering of Spearman correlation coefficients for 16 A. niger ATAC-seq datasets

nucleic acid bands (Fig. 1b). The insert size distribution of sequenced fragments from A. niger chromatin had a clear periodicity of approximately 200 bp (Fig. 1c, Additional file 1: Figure S1), suggesting many fragments are protected by integer multiples of nucleosomes.

We analyzed the pattern of mapped ATAC-seq reads around the transcription start site (TSS) in A. niger
CBS513.88. Heatmaps showed the intensity of mapped ATAC-seq reads flanking 1-kb upstream and downstream of annotated TSSs in the AspGD database (Fig. 1d). Furthermore, separate heatmaps showed that fragments less than $100 \mathrm{bp}$ clustered immediately upstream of TSSs throughout the A. niger genome. Fragments between 180 and $247 \mathrm{bp}$, corresponding to nucleosome 
footprints, were depleted from TSSs throughout the genome. Overall, the identified accessible regions were mostly enriched around the TSSs (Fig. 1d), which is consistent with the fact that these regions usually contain active regulatory cis-elements. The signal intensity of accessible regions showed a clear depletion and periodic peaks as the distance from the TSS (Fig. 1e), which is consistent with the presence of a positioned nucleosome +1 that serves as barrier, dictating the positioning of neighboring nucleosomes (especially nucleosome -1 ). This phenomenon attenuates at further distances from the barrier [34]. Furthermore, using ATAC-seq for $A$. niger, we generated paired-end ATAC-seq libraries for 16 samples, and the libraries ranged from 35.9 to 104.9 million reads (Additional file 2: Table S1). The percentages of reads in two replicate samples of CBS513.88 WT1 and CBS513.88_WT2 that aligned to the A. niger CBS513.88 genome was $84.23 \%$ and $86.31 \%$, respectively (Additional file 2: Table S1). The insert size distributions of sequenced fragments from 16 ATAC-seq libraries of $A$. niger had a similar periodicity (Additional file 1: Figure S1). Spearman correlation analysis was used to inspect the reproducibility of biological and technical replicates. Heatmap clustering of Spearman correlation coefficients for $16 \mathrm{~A}$. niger ATAC-seq datasets revealed a strong correlation between replicates of the same strain or the mutant and a lower correlation between different strains (Fig. 1f). Taken together, these data suggested that the ATAC-seq protocol of filamentous fungus $A$. niger could provide data on accessible regions of chromatin in a genome-wide search for regulatory elements.

Identification of genome-wide chromatin accessibility and comparison of open chromatin profiles among Aspergillus species and strains

Visualization of the A. niger CBS513.88 and SH2 ATACseq data sets on a genome scale revealed that they were similar to each other, and the Tn5 integration regions of naked genomic DNA were uniformly distributed along the entire chromosome (Fig. 2a). The syntenic analysis showed that most of the genomic sequences were homologous between $A$. niger strains CBS513.88 and SH2, except for a missing DNA fragment in $A$. niger CBS513.88 contig VIII_An18 and a fragment in A. niger SH2 contig S6 (Fig. 2a). A total of 7297 and 7697 accessible chromatin regions (peaks) were identified in A. niger CBS513.88 and SH2, respectively (Fig. 2b and Additional file 3: Table S2). Among these accessible regions, 6524 regions overlapped, indicating that these regions contained common cis-elements for both strains. These common regulatory elements might play a basal transcriptional regulatory role in $A$. niger species. Additionally, 773 and 1173 accessible regions are specific for $A$. niger CBS513.88 and SH2, respectively, and these accessible regions contained differential cis-elements for each strain. A heatmap of the peak signal for the 2-kb flanking region of the peak center (Fig. 2c) showed that most of the accessible regions formed sharp peaks in the center and ranged from narrow to wide, which provided valuable data depending on cis-element motifs in these accessible regions. On the genome scale, accessible regions are highly enriched in promoter regions (Fig. 2d), which was consistent with the distribution of accessible regions in Fig. 1d. In addition to promoter regions, the transcription terminator site (TTS) regions contain the maximal accessible regions $(12.88 \%$ for CBS513.88 and $15.70 \%$ for $\mathrm{SH} 2$, Fig. 2d). These accessible regions in TTSs indicated that gene downstream regions also contain regulatory elements for gene transcription.

As the GRAS strains, $A$. niger and $A$. oryzae share the common characteristics for the production of heterologous protein, protease, and natural products. To understand the syntenic orthologues of accessible regions across the Aspergillus species, we compared the A. niger CBS513.88 and A. oryzae niaD300 ATAC-seq peaks on a genome-wide scale. Our results showed that these two Aspergillus species had strong peak synteny, and there were 1564 syntenic orthologues of ATAC-seq peaks identified between $A$. niger CBS513.88 and A. oryzae niaD300 (Fig. 2e, Additional file 4: Table S3). Then, we located the target genes of these syntenic peaks and analyzed their GO enrichment function (Fig. 2f). Ribosomes related to GO categories were significantly enriched in these target genes, especially for the following three categories: "structural constituent of ribosome (MF)," "ribosome (CC)," and "translation (BP)." These highly enriched target genes indicated that Aspergillus species strains possessed the highly homologous regulatory mechanisms in terms of protein synthesis. Therefore, ATAC-seq data can be used for comparative genome analysis to explain the conservation of the evolutionary process of organisms at the genome level.

\section{Identification of significantly changed chromatin accessibility driven by transcription factors and chromosome regulatory factor in Aspergillus species}

A clustering map of ATAC-seq peaks for 16 A. niger samples indicates that there were common ATAC-seq patterns among all $A$. niger strains and specifically showed that $\mathrm{SH} 2$ and its derivatives did not have ATAC-seq signals corresponding to the 200-kb missing genomic DNA fragments (Fig. 3a) [4]. Furthermore, we identified the ATAC-seq peaks that significantly changed accessibility (FDR $<0.05)$ among $A$. niger $\mathrm{SH} 2$ cultured under different conditions and its TF-deleted strains (Fig. 3b and Additional file 5: Table S4). A total of 4009 of the differential ATAC-seq peaks (DAPs) in the 

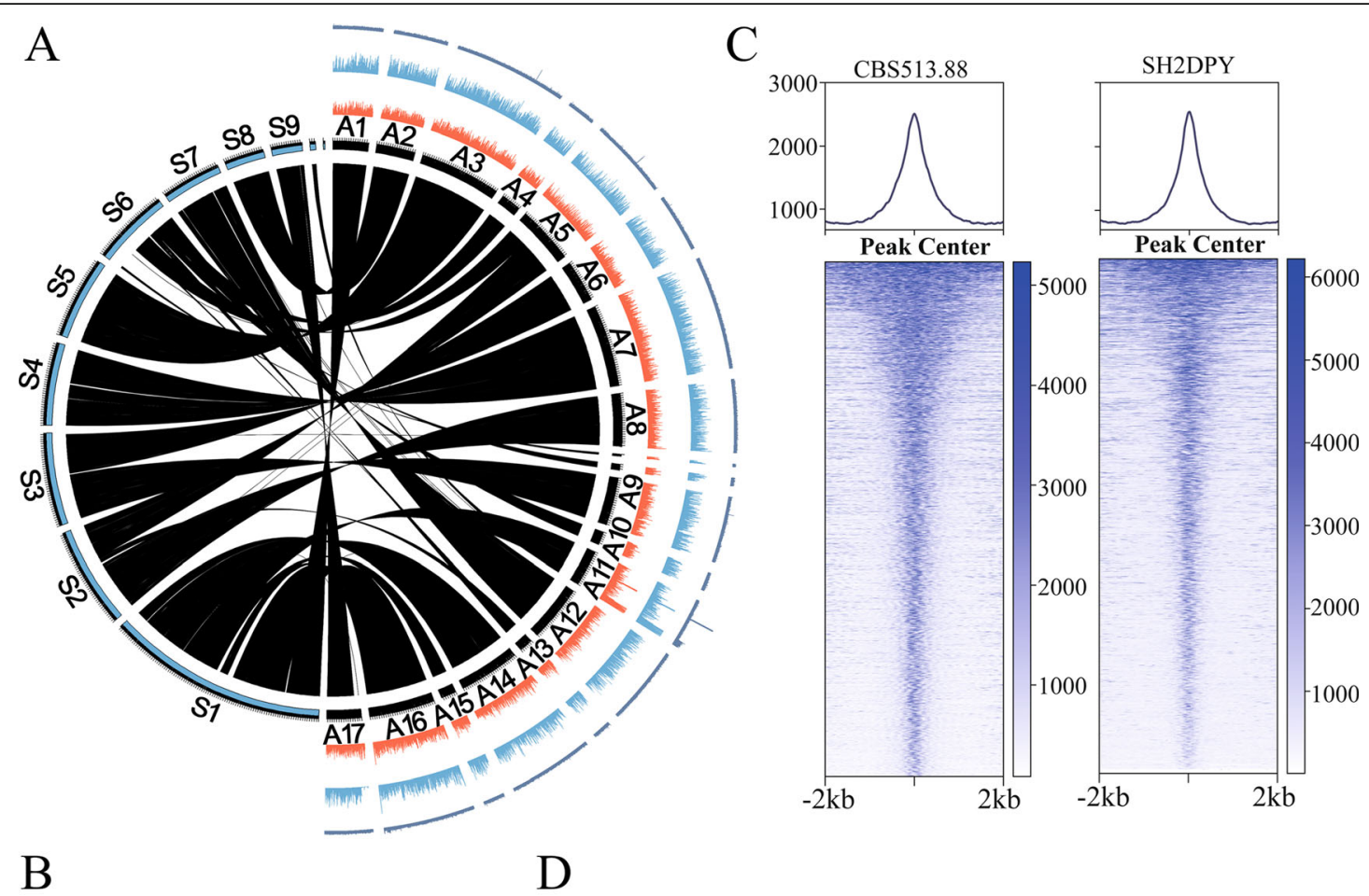

B
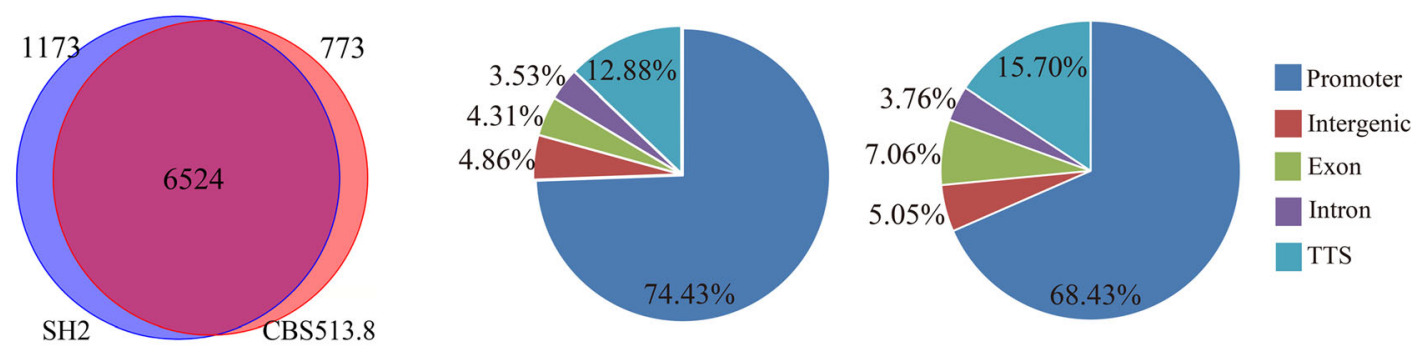

E

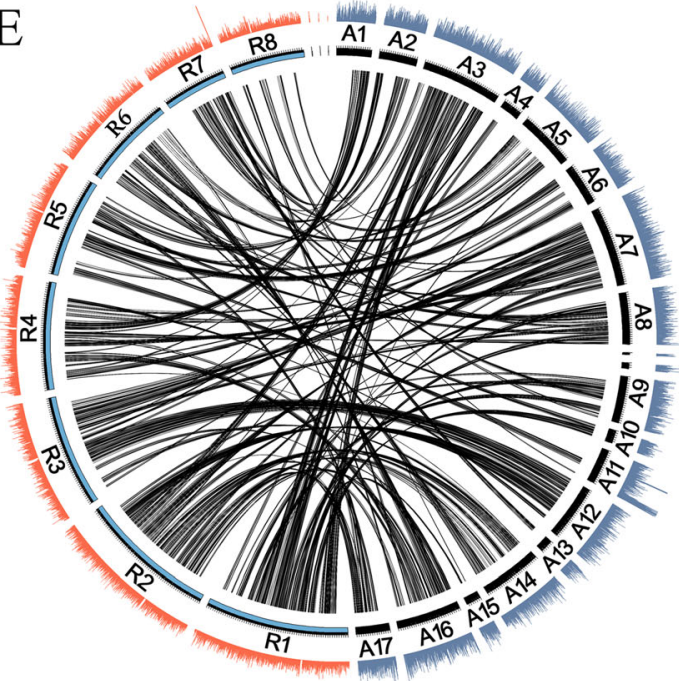

F

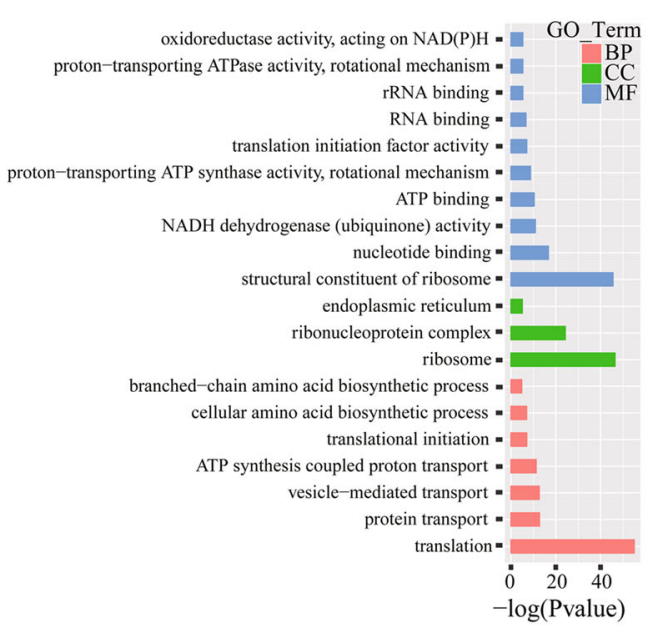

Fig. 2 (See legend on next page.) 
(See figure on previous page.)

Fig. 2 Whole-genome landscape of chromatin accessibility in A. niger. a Syntenic analysis between A. niger CBS513.88 (contig A1-A17 corresponding to An01-An19) and SH2 (contig S1-S11), and visualization of ATAC-seq peak signals in A. niger CBS513.88 (orange) and SH2 (blue). The outer signal represents the A. niger CBS513.88 naked genomic DNA which was used as the integration control of Tn5 transposase. $\mathbf{b}$ A Venn diagram showing the overlap of accessible regions between A. niger CBS513.88 and SH2. c Density plots of mapped reads (upper) and heatmap of peak length (bottom) for the 2-kb region flanking the peak center. $\mathbf{d}$ Distribution of accessible regions in the promoter, intergenic region, exon, intron, and TTS in A. niger CBS513.88 and SH2. e Syntenic analysis of ATAC-seq peaks between A. niger CBS513.88 (contig A1-A17) and A. oryzae niaD30 (contig R1-R8). f Functional clustering of overlapping ATAC-seq peaks of A. niger CBS513.88 and A. oryzae niaD300

promoter regions were identified when comparing with A. niger SH2 cultured in DPY and MPY media (Additional file 5: Table S4). We found that changing the culture conditions could slightly alter the chromatin accessibility, in which 3853 of the DAPs were specific to A. niger $\mathrm{SH} 2$ cultured under MPY medium (Additional file 5: Table S4). Almost all upregulated genes were driven by the MPY-specific ATAC-seq peaks (Fig. 3b: MPY_vs_DPY). Besides, the induced conditions such as alternative carbon sources or differential $\mathrm{pH}$ could also slightly alter the chromatin accessibility (Data submitted to NCBI, PRJNA692847), and low-level CreA can strongly inhibit the expression of $x \ln D$ and $a b f A$ (Additional file 1: Figure S2). Furthermore, we identified TFspecific chromatin accessibility using the peak signal intensity of $A$. niger $\mathrm{SH} 2$ compared with that observed in TF-deleted strains (FDR <0.05). In total, 1641 of the amyR-specific peaks, 1156 of the prtT-specific peaks,
773 of the cpcA-specific peaks, 1301 of the pacC-specific peaks, and 1305 of the creA-specific peaks in the promoter regions exhibited significantly higher accessibility $($ FDR $<0.05)$ than those in the corresponding TFdeleted strains (Additional file 5: Table S4). The distributions of upregulated genes were unbalanced and trended towards TF-specific chromatin accessibility (Fig. $3 b)$.

To confirm the ATAC-seq data, we performed ChIPseq of the protease regulator PrtT (NCBI accession NO.: PRJNA692789). A total of 161 specific peaks were enriched, of which 97 (60.24\%) peaks could be mapped with delprtT and 112 (69.57\%) peaks with WT (Fig. 3c). The conserved motif CCGHCGG [35] could also been enriched (Additional file 1: Figure S3). Furthermore, the coupling analysis of ChIP-seq, RNA-seq, and ATAC-seq data of PrtT (Fig. 3d-f and Additional file 1: Figure S4) showed three major regulation categories of TF: (i) the

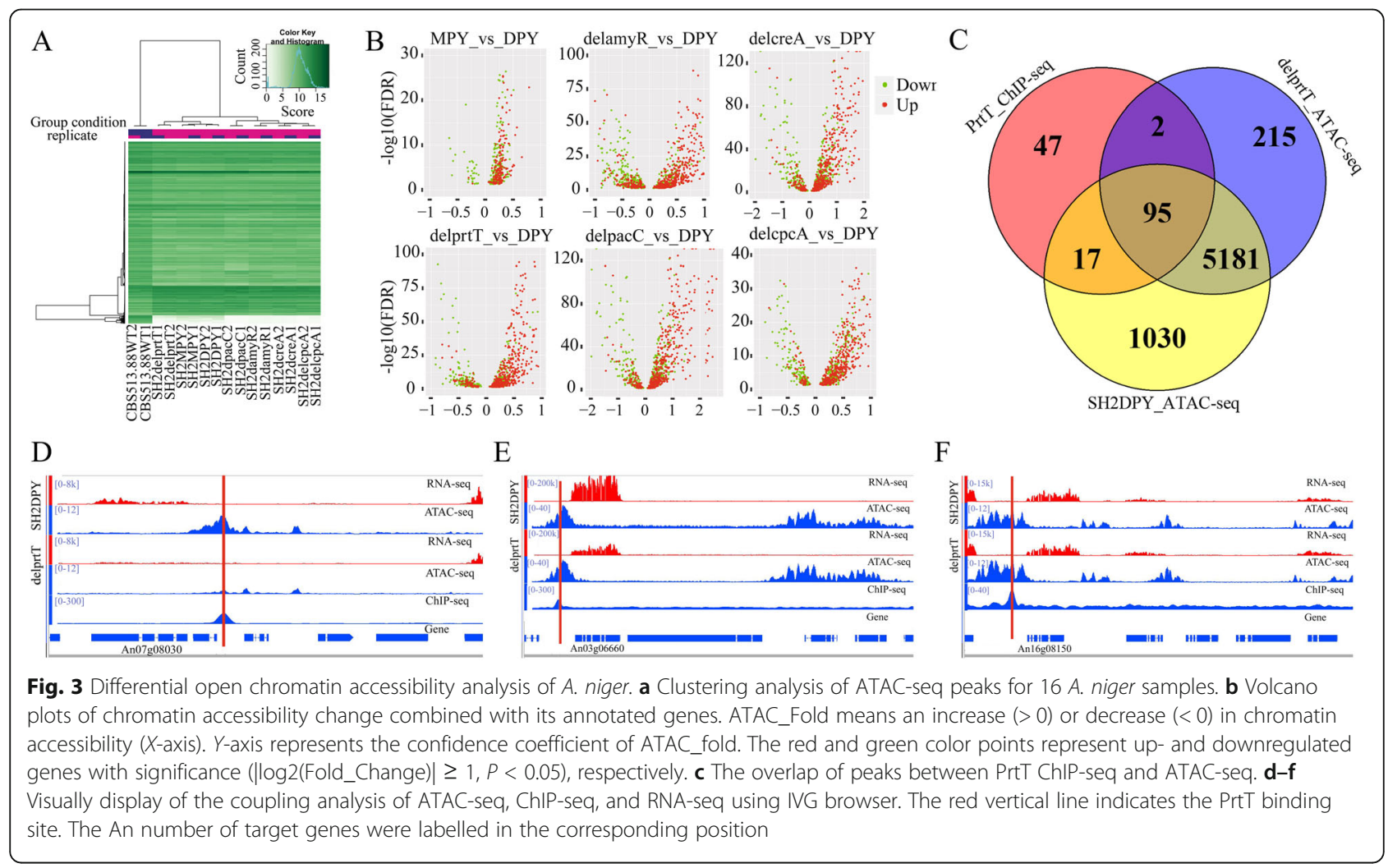


deletion of PrtT leads to decreased chromatin accessibility, thereby downregulating target genes (Fig. 3d and Additional file 1: Figure S4a); (ii) the deletion of PrtT does not affect chromatin accessibility, but downregulates target genes (Fig. 3e and Additional file 1: Figure S4b); (iii) the deletion of PrtT neither affects chromatin accessibility, nor affects gene expression (Fig. 3f and Additional file 1: Figure S4c).

The biosynthetic gene clusters of secondary metabolites (SMs) in Aspergillus species are frequently silent or inactive under normal laboratory conditions due to a tendency to be located in subtelomeric regions with a high degree of heterochromatin condition; however, these genes can be activated by changing the chromosomal state of the SM gene clusters from heterochromatin to euchromatin [36]. LaeA has been acknowledged as a global regulator of secondary metabolism in Aspergilli. Deletion of LaeA in filamentous fungi decreased the virulence of the strains and the production of secondary metabolites. And it has been reported that deletion or overexpression of LaeA could be used to explore numerous secondary metabolite clusters [37-39], although the physiological function of LaeA in modulating gene expression remains little known [40]. To investigate whether LaeA could regulate the chromatin accessibility in Aspergilli, we constructed ATAC-seq libraries of dellaeA and OElaeA and identified the ATAC-seq peaks with significantly changed accessibility $(F D R<0.05)$ in dellaeA-vs-WT (4725 DAPs) and OElaeA-vs-WT (5572 DAPs). (Figure 4a, Additional file 1: Figure S5; Additional file 2: Table S1, and Additional file 6: Table S5). SM biosynthetic gene clusters in the A. oryzae RIB40 genome contain 621 biosynthetic SM genes predicted by Secondary Metabolite Unique Regions Finder (SMURF) [41]. The chromatin regions in front of 138 and 164 of the SM biosynthetic genes in A. oryzae OElaeA and dellaeA mutants significantly changed accessibility (FDR < 0.05 ) to induce significant regulation of 42 and $32 \mathrm{SM}$ biosynthetic genes, respectively (Fig. 4a and Additional file 6: Table S5). The 54 backbone genes of SM biosynthesis include polyketide synthases (PKSs) and nonribosomal peptide synthetases (NRPSs) from the A. oryzae RIB40 genome; only 12 backbone genes of SM biosynthesis significantly changed accessibility $($ FDR $<0.05)$ to induce significant regulation of the corresponding SM backbone genes in OElaeA and dellaeA mutants, which contained 7 NRPSs, 4 PKSs, and 1 DMAT (Additional file 6: Table S5). We found that the knockout and overexpression of the laeA gene only up- and downregulated the SM biosynthetic genes that were expressed in the wild-type strain, while there was never de novo activation of those SM biosynthetic genes that were not expressed in the wild-type strain (Additional file 1: Figure S6). Furthermore, we investigated chromatin accessibility and transcriptional activity in the famous aflatoxin (AF) biosynthesis pathway of A. oryzae (Fig. 4b). The sequences of the aflatoxin (AF) biosynthesis gene clusters were divided into two parts: active and inactive chromatin regions. The sequences from AO090026000004 to AO090026000020 were the active chromatin regions, which were characterized by highly expressed biosynthesis genes. The overexpression and knockout of the laeA gene were able to change the chromatin accessibility in the active regions. The key transcription factor aflR (AO090026000014) was in the region of high chromatin accessibility, while the coactivator aflJ (AO090026000015) became highly expressed following the overexpression of laeA. The other sequences from AO090026000020 to AO090026000031 were in inactive chromatin regions in which no ATACseq peaks were identified, and biosynthesis genes had no expression or the lowest level of expression. The AF biosynthesis pathway components were located in a laeAdependent SM biosynthetic gene cluster. Taken together, the reason for the lack of AF biosynthesis in A. oryzae might be inactive chromatin regions around the AF biosynthesis pathway.

\section{Identification of footprints in filamentous fungus Aspergillus niger}

DNA regions of footprints bounded by DNA-binding proteins are protected from $\operatorname{Tn} 5$ integration, at which the read coverage suddenly drops [26, 42]. A high-depth ATAC-seq can be used to identify depleted narrow regions in open chromatin regions of a genome corresponding to a single TF footprint [26, 42, 43]. We used the "wellington_footprints.py" function of the pyDNase package [44] to systematically identify $\operatorname{Tn} 5$ integrationinsensitive sites from CBS513.88 and $\mathrm{SH} 2$ and footprints from ATAC-seq peaks (Fig. 5a). In total, there were 24,925 and 22,548 DNA footprints detected in vivo across the A. niger genome from 7297 and 7697 ATACseq peaks of CBS513.88 and SH2, respectively (footprint data from all wild-type and TF-deleted strains can be found in Additional file 7: Table S6). A total of 14,176 DNA footprints overlapped between CBS513.88 and SH2 ATAC-seq data. The DNA footprints identified by the ATAC-seq data were enriched 1-kb upstream and 0.5-kb downstream of the TSSs (Fig. 5b). To further characterize genomic footprints, we performed de novo motif enrichment analysis on the footprints from CBS513.88 and SH2 using the "findMotifsGenome.pl" function of the HOMER package with the default settings [45]. A total of 15 and 17 de novo TFBs with a $P$ value threshold of 1.0E-12 were enriched more than 5fold from the two sets of footprint data from CBS513.88 and $\mathrm{SH} 2$, respectively (Fig. $5 \mathrm{c}$ and Additional file 8: Table S7). De novo TFBs of other SH2 TF-deleted 


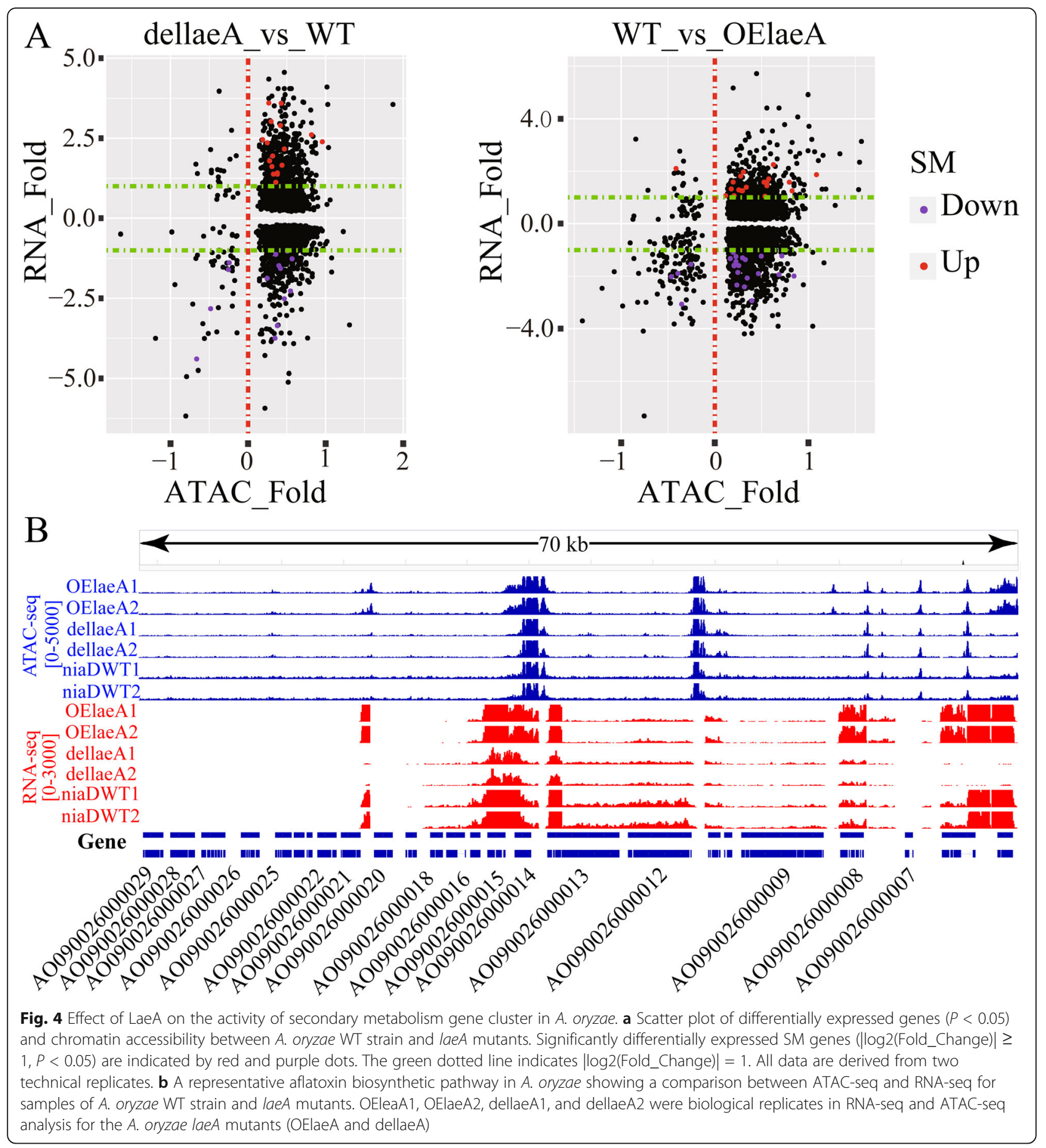

strains with a $P$ value threshold of $1 \mathrm{E}-50$ were also identified using the HOMER method (Additional file 1: Figure S7 and Additional file 8: Table S7). The E-box of bHLH factors (motif TCACGTGATC), the SltA homolog binding motif (motif CTGCCTGAGGCA), and the core element of bZIP factors (motif GCTGAGTCAGCV ) were among the top 5 most enriched motifs according to $P$ value in all de novo TF binding motifs (Fig. $5 \mathrm{c}$ ).
Instances of each TF binding motif were identified using the "annotatePeaks.pl - $\mathrm{m}$ " function of the HOMER package to search for DNA sequences in the 24,925 DNA footprints from A. niger CBS513.88 and the 22,548 DNA footprints from $A$. niger SH2 (Additional file 9: Table S8). Instances of genes controlled by de novo TF binding at motifs were identified in footprints in the promoter regions, and they were analyzed for the 

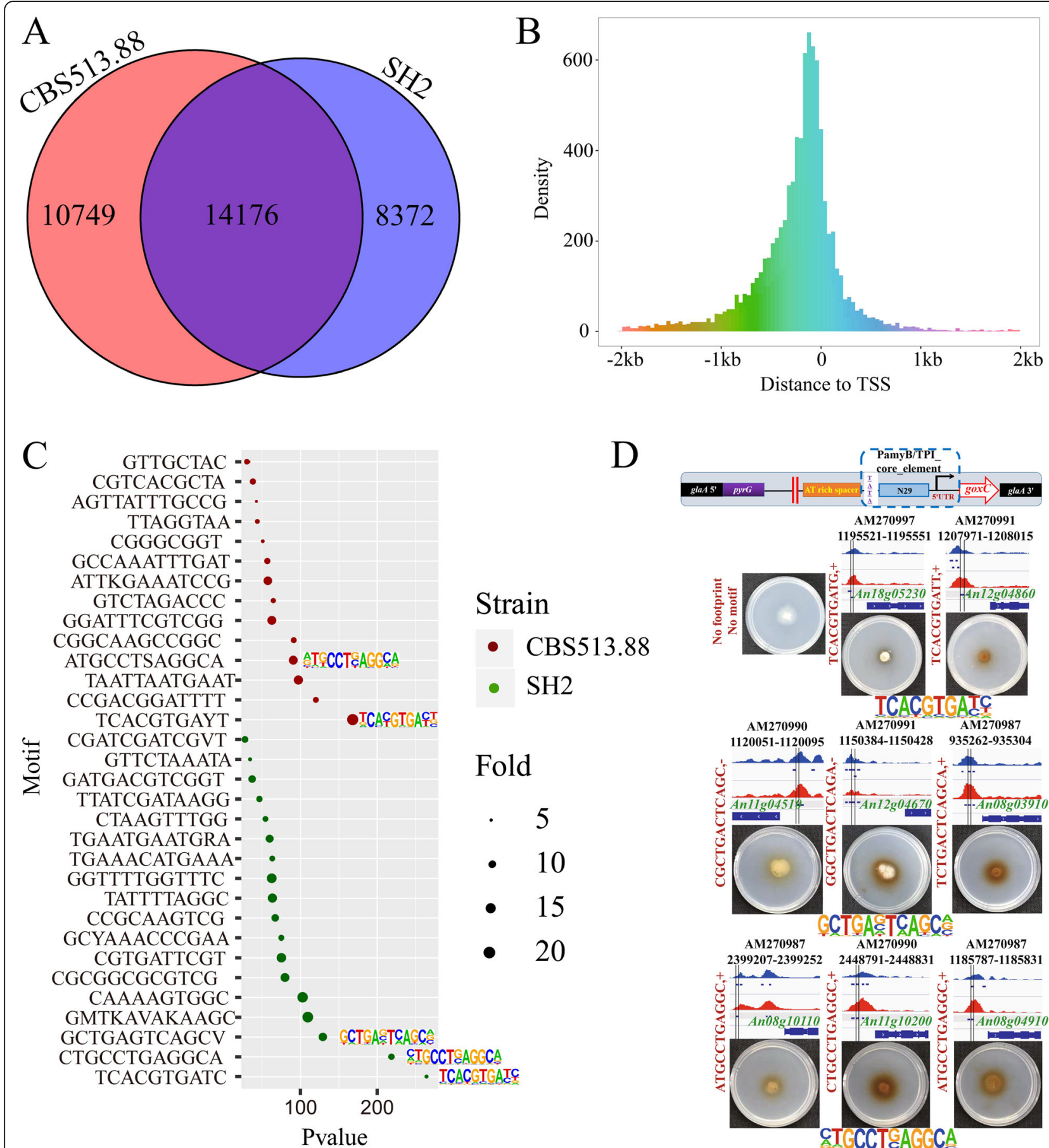

$\mathrm{D}$

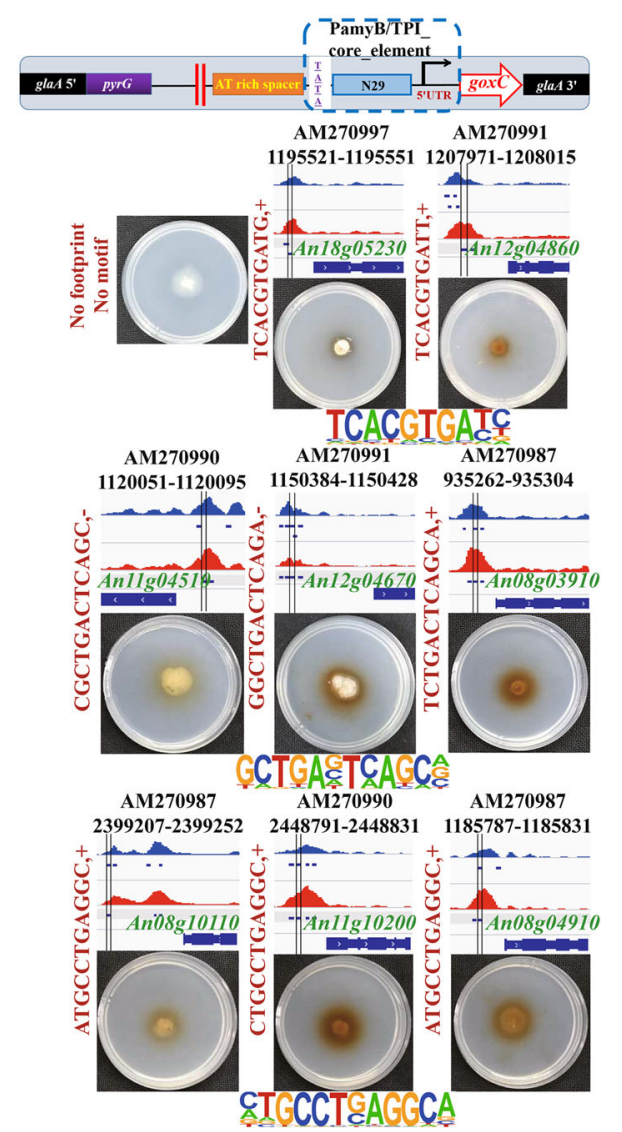

Fig. 5 Identification of de novo predicted footprints in A. niger CBS513.88 and SH2, and functional verification in regulating gene expression. a Venn diagram analysis of identified footprints in A. niger CBS513.88 and SH2. b Statistical analysis of the distances between the identified DNA footprints and the TSSs. c De novo prediction of transcription factors in A. niger CBS513.88 (red) and SH2 (green). The circle size represents the enrichment fold. The top three transcription factor motifs in $\mathrm{A}$. niger $\mathrm{SH} 2$ are labeled with the corresponding binding motifs. The $x$-axis represents $P$ value. $\mathbf{d}$ In vivo functional verification of putative de novo transcription factor targeting sites were carried out by artificially synthesized minimal promoters. The red dividing line represents the location of the footprints to be verified. The red text on the side is the TF binding motif contained in the verified DNA instances. From top to bottom, there were 2 E-box of bHLH factors (motif TCACGTGATC), 3 core elements of bZIP factors and 3 SltA homologs binding motif (motif CTGCCTGAGGCA) target sites validated by phenotype plates in vivo 
functional enrichment of corresponding TF binding motifs (Additional file 1: Figure S8). In the A. niger SH2, 269 genes under the control of the de novo motif TCAC GTGATC (E-box) were enriched in GO categories "structural constituent of the ribosome" and "translation" (Additional file 1: Figure S8a). In addition, 154 genes under the control of the de novo motif CTGC CTGAGGCA (SltA homolog) were enriched in the molecular function GO categories "ATP binding" and "structural constituent of the ribosome;" in the cellular components of "ribosome," "mitochondrial inner membrane," and "vacuole;"; and for the biological processes of "translation" and "proteolysis" (Additional file 1: Figure S8b). Then, 54 genes under the control of the de novo motif GCTGAGTCAGCV (bZIP factors) were also enriched in the molecular function GO categories "zinc ion binding," "DNA binding," and "transcription factor activity" in the nucleus for the process of regulation of transcription (Additional file 1: Figure S8c).

Furthermore, to detect whether the ATAC-seq footprints containing the TF binding motifs drove the expression of functional target genes, we constructed a minimal synthetic promoter consisting of a core element, an ATAT-binding box, an AT-rich spacer, and a footprint library-driven UAS element with a TF binding site motif (Additional file 1: Figure S9) [46]. A 29-bp sequence from the TSS in the $a m y B$ (An05g02100) promoter of $A$. niger was chosen as the core element due to the high-level expression of the $a m y B$ gene. The glucose oxidase goxC (An01g14740) was used as a reporter, the expression of which would be revealed by a colored colony. The repression under ER stress (RESS) element identified in our prior study [46] was used as a positive control, and it also produces colored colonies (Additional file 1: Figure S9). As a negative control, a sequence containing only the $a m y B$ core element, the ATAT sequence, and the AT-rich spacer was constructed (no colored colonies but basal transcription can occur (Additional file 1: Figure S9)). We used this minimal promoter system to determine whether the ATACseq footprints containing de novo motifs, such as motif TCACGTGATC (E-box), motif CTGCCTGAGGCA (SltA homolog), and motif GCTGAGTCAGCV (bZIP factors), could drive the expression of target genes (Fig. $5 \mathrm{~d})$. Verifying the translation and ribosome functions controlled by the motif TCACGTGATC (E-box), the footprint of An12g04860, which encodes a ribosomal protein of the large subunit L30, was found to drive the expression of the reporter gene in vivo, resulting in colored colonies (Fig. 5d). The footprints containing motif CTGCCTGAGGCA located in the promoter regions of An08g04910 and An11g10200, which encode the
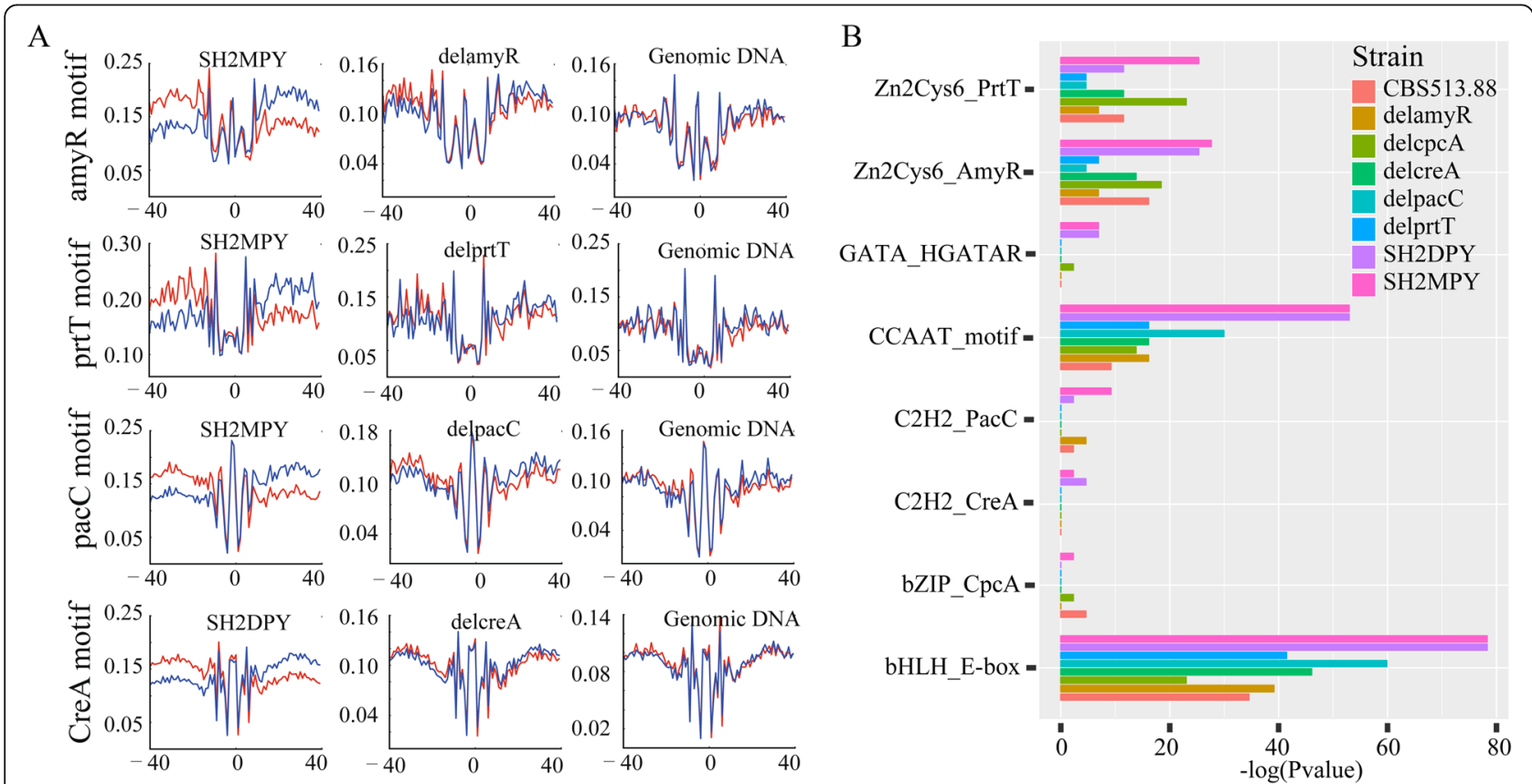

Fig. 6 Distribution of Tn5 integration around known footprints and enrichment analysis of known TF motifs. a Distribution of Tn5 integration sites in the ATAC-seq data of SH2MPY (left), TF deletion strains (middle), and naked genomic DNA (right) around known motifs including amyR, prtT, pacC, and creA. Tn5 integration sites showed similar patterns around known TF motifs between TF deletion strains and naked genomic DNA whereas the patterns are different for SH2MPY. The red line indicates the integration sites on the positive-sense strand whereas the blue line indicates those on the anti-sense strand. Y-axis represents average Tn5 integrations. The frequency analysis was carried out by combining two technical replicates. b Enrichment analysis of known TF motifs in A. niger CBS513.88, SH2, and TFs deletion mutants; - $\log (P$ value) was used as a significant criterion 
$\mathrm{NADH}$-ubiquinone oxidoreductase and cytochrome $\mathrm{C}$ oxidase subunit in the electron respiratory chain, respectively, also drove the expression of the reporter gene in vivo. The footprints containing the motif GCTGAG TCAGCV, which is located in the promoter regions of An11g04510 and An12g04670 (related to translation release factor activity and translation initiation factor, respectively), were confirmed to drive the expression of the reporter gene in vivo. Taken together, genome-wide DNA footprints were systematically identified from accessible chromatin regions based on our ATAC-seq data. In addition, de novo motifs enriched from ATAC-seq footprints were able to drive the expression of their target genes in vivo.

\section{Known TF motifs of Aspergillus niger enrichment in ATAC- seq footprints}

We determined the transposase Tn5 integration patterns around the binding sites of AmyR, PrtT, PacC, and CreA in the $A$. niger $\mathrm{SH} 2$ cultured under MPY medium (Fig. 6a), while these patterns for known TFs were also evident in naked genomic DNA (Fig. 6a). We further found that distributions of Tn5 integration sites in the flanking regions of binding site motif sequences were asymmetrical when comparing the anti-sense and sense strand and the patterns which were completely different from the symmetrical patterns observed in the naked genomic DNA. Furthermore, to elucidate whether strand-specific Tn5 integration patterns around TF binding sites are caused by protein/DNA interactions, we calculated the Tn5 integration frequency in TF-deleted strains (Fig. 6a). In this experiment, the conserved DNA motif should not be enriched, for there was no corresponding known TF to be expressed in vivo. Tn5 integration sites around the known TF binding sites in the absence of the corresponding TF condition showed a similar pattern to that of genomic DNA, which was a symmetrical integration pattern around TF binding sites on both DNA strands. The asymmetrical pattern of Tn5 integration around TF binding sites in our ATAC-seq data was distinctly different from the no TF binding pattern on both TF-deleted strains in vivo and naked genomic DNA in vitro (Fig. 6a). We demonstrated here that strand-specific Tn5 integration patterns around TF binding sites are caused by protein/DNA interactions, which could be used as a signal indicating an active TFBs.

To determine the enrichment of known TF binding motifs from ATAC-seq footprints by comparing ATACseq data from $A$. niger WT strain and its TF-deleted strains, we collected the available binding motif sequences of 19 known Aspergillus TFs [23] and employed the "findMotifsGenome.pl" function of the HOMER package to identify overrepresented motifs of known Aspergillus TFs from ATAC-seq footprint data. We found that the binding motifs of bZIP CpcA, C2H2 CreA, and PacC were not overrepresented in the ATAC-seq footprints of the corresponding TF-deleted strains ( $P$ value $>1.0 \mathrm{E}-2$ ) (Fig. 6b). Although the binding motifs of the $\mathrm{Zn}_{2} \mathrm{Cys}_{6}$ family AmyR and PrtT were also enriched in the footprints from the TF-deleted strains delamyR and delprtT, respectively, the enrichment $P$ values of AmyR and PrtT motifs were less than 1.0E-2 (Fig. 6b). The binding motifs for bHLH family E-box, CCAAT binding complex (CBC), $\mathrm{Zn}_{2} \mathrm{Cys}_{6}$ family AmyR, and PrtT were significantly enriched in all $A$. niger strains, especially the overrepresentative enrichment of $\mathrm{SH} 2$ in MPY medium (Fig. 6b). Other TF family motifs, such as bZip CpcA, GATA AreA, C2H2 CreA, and PacC, were only enriched in the ATAC-seq footprints of the $\mathrm{SH} 2$ cultured in MPY and DPY medium (Fig. 6b). Footprints that contained a significant motif match were considered to be instances of predicted binding motifs. We used the "annotatePeaks.pl -m" function of the HOMER package to detect the motif instances of known TFs from footprints by comparing $A$. niger WT strains and the corresponding TF-deleted strains (Additional file 10: Table S9). The motifs of AmyR, PrtT, PacC, and CreA were identified in $765,158,821$, and 2865 motif instances in the $\mathrm{SH} 2$ cultured in MPY, respectively (Additional file 10: Table S9). However, we still detected 593 instances of AmyR binding, 103 instances of PrtT binding, 656 instances of PacC binding, and 2648 instances of CreA binding from footprints in the TF-deleted strains delamyR, delprt $\mathrm{T}$, delpacC, and delcreA, respectively (Additional file 10: Table S9).

Furthermore, to investigate whether instances of TF binding drove the expression of target genes, we used TF binding motif sites in ATAC-seq footprints combined with RNA-seq data for the changes in gene expression between the $\mathrm{SH} 2$ cultured in MPY and the corresponding TF-deleted strains (Fig. 7a). The expression of 1248 genes in the delamyR and 1242 genes in the delprtT was downregulated compared to what was seen in the control of the SH2 strain (Fig. 7a). Only 78 and 23 genes were downregulated in the delamyR and delprtT strains overlapped with motif target genes, respectively (Fig. 7a). As expected, 23 target genes under the control of PrtT were mainly involved in serine-type peptidase activity, and 78 target genes under the control of AmyR were functionally enriched in hydrolase activity, acting on glycosyl bonds (Fig. 7a). A total of 1644 genes were upregulated in the delcreA compared with the control of the SH2 strain, in which 269 genes overlapped with motif target genes from CreA (Fig. 7a). GO functional enrichment analysis revealed that 269 genes under the control of CreA were primarily involved in the molecular function of hydrolase activity and transporter activity. Besides, the enriched genes were 


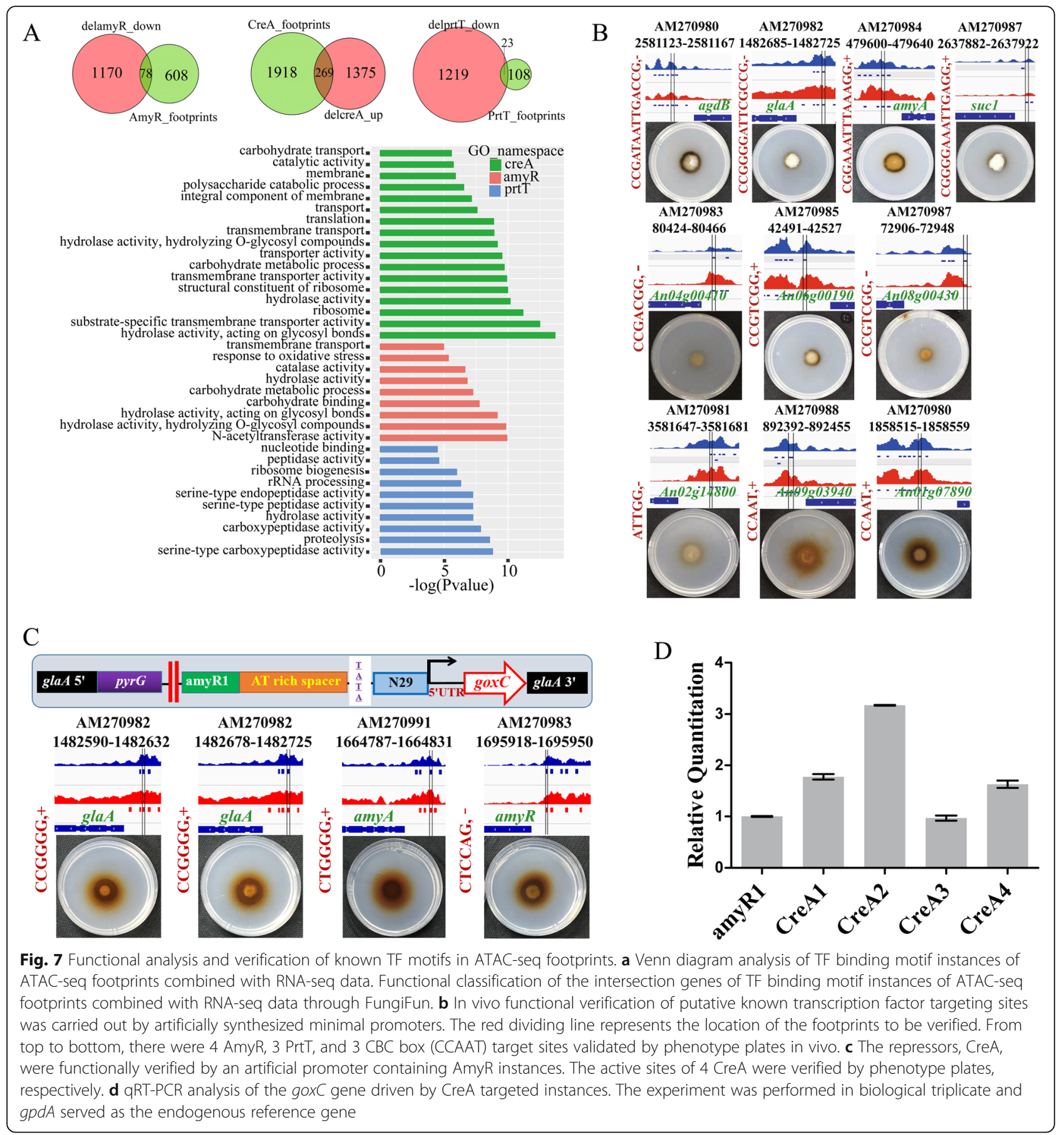

clustered in the structural constituents of the ribosome, the cellular components of membranes and ribosomes, and their biological processes involved in carbohydrate catabolism, carbohydrate transport, and translation (Fig. 7a).

To experimentally assess whether footprints with these known TF binding motifs that were identified by ATACseq data were functional TF binding sites, we performed footprint-driven reporter assays in vivo (Fig. $7 b$ ). Three footprints containing the AmyR binding motif were located in promoters of glycosyl bond hydrolase genes: An01g10930 (agdB), An03g06550 (glaA), and An05g02100 (amyA). These AmyR binding motifs drove the expression of the reporter gene, resulting in colored colonies. Three footprints containing the PrtT binding motif in the promoter of peptidase genes, including An04g00410 (dipeptidyl peptidase III), An06g00190 (Sedolisin family secreted protease), and An08g00430 
(serine-type carboxypeptidase), also drove the expression of the reporter gene. In addition, footprints containing CCAAT box or AreA binding motifs can also function as transcription activator (Fig. $7 \mathrm{~b}$ and Additional file 1: Figure S10).

In addition to identifying the activation function of ATAC-seq footprints containing the known TF binding motifs, we further investigated whether ATAC-seq footprints containing repressor binding motifs could downregulate the expression of target genes (Fig. 7c). We constructed a minimal synthetic promoter with the footprint-driven UAS elements containing the AmyR binding motif instance (An01g10930, $a g d B$ ) as a positive control (Fig. 7c). The footprint containing the repressor binding motif was assessed by assembling downstream of the AmyR footprint. A. niger CreA (An02g03830, contains two $\mathrm{Cys}_{2} \mathrm{His}_{2}$ zinc finger motifs) is the transcriptional regulator that mediates carbon catabolite repression [47]. However, our results showed that footprints containing CreA binding motif sites located in promoters of glycosyl bond hydrolases such as gla $A$ (An03g06550), amyA (An12g06930), and $a m y R$ (An04g06910) were able to upregulate the expression of the reporter gene (Fig. 7c, d). Two footprints containing the CreA binding motif in the glaA promoter possessed the same ability to activate functional gene expression (Fig. 7c). Taken together, the transcriptional regulator CreA acts not only as repressor but also as activator.

\section{Discussion}

In ATAC-seq, the Tn5 transposase integrates its adaptor payload into locations of open chromatin, which can be amplified by PCR and assessed with high-throughput sequencing to study protein/DNA interactions on a genome scale [27]. Here, we developed an ATAC-seq protocol for filamentous fungi to identify chromatin accessibility on a genome scale. Our initial attempts failed to generate ATAC-seq data from the chromatin following digestion of $\operatorname{Tn} 5$ transposase, harvesting the mycelium and reducing it to powder by grinding it in liquid nitrogen with a mortar and pestle. The gel image analysis shows no discrete nucleic acid bands, and this method resulted in a high noise-to-signal ratio after sequencing. In our modified Aspergillus ATAC-seq protocol, the lysis of the Aspergilli cell wall was carried out by adding hydrolase during cultivation, and protoplasts were obtained by filtration through a miracloth membrane. High-quality intact nuclei released from A. niger protoplasts, which are similar to mammalian cells, were used to improve the data collected from ATAC-seq. ATAC-seq requires few cells, so a sufficient number of protoplasts were easily obtained from $A$. niger cultured under various conditions. Yeast spheroplasts from S. cerevisiae and Schizosaccharomyces pombe have also been used in ATAC-seq assays [33]. Furthermore, protoplast preparation could occur with $A$. niger cells cultured under various conditions to decrease experimental variation. In our $A$. niger ATAC-seq library, we detected the same periodicity pattern in the insert size distribution of sequenced fragments from chromatin as what was observed in a human lymphoblastoid cell line [27] and in Arabidopsis thaliana [43]. The A. niger ATAC-seq library has a high signal-to-noise ratio, which was controlled by comparing the data to Tn 5 integration into naked genomic DNA. The reproducibility of biological and technical replicates in our A. niger ATAC-seq protocol was relatively high. In ATAC-seq experiments using mammalian cells and plant cells, typically $\sim 30-70 \%$ of the sequenced reads are aligned to the organellar genomes [27, 43]. In our A. niger CBS513.88 ATAC-seq results, a significantly higher fraction of reads were mapped to the nuclear genome. These data show that our Aspergillus ATAC-seq protocol is an effective method that can be applied to the analysis of other species of filamentous fungi.

The chromatin accessibility regions of $A$. niger CBS513.88 and SH2 were similarly distributed across the various elements of the genome and showed a high proportion at TSSs $(\sim 70 \%)$ and a low proportion at intergenic regions $(\sim 5 \%)$, which was contrary to humans [48], Arabidopsis [32], and tomato [8]. This difference may reflect the smaller size of the A. niger genome (35 $\mathrm{Mb})$ [3] compared with the larger and less compact genomes of humans and plants. In addition, TTS also contained a small number of accessibility regions $(\sim 13 \%)$, which is similar to what was observed in the mouse [28]. Therefore, we reasoned that TTSs may also play an important role in the transcriptional regulation of genes and downstream translational control in A. niger.

Prior works found that the accessible chromatin regions were positively correlated with gene expression changes of the nearest target genes [28, 32, 49]. However, the regulatory regions could also be suppressed by some TFs or nucleosomes [49]. Our results further verified the coexistence of transcriptional activation and inhibition in the accessible region. Besides, the gene transcription activation can be accomplished by a single TF alone, or by a combination of multiple TFs (Fig. 3). Under the condition of single activation, TF-deleted directly affects chromatin accessibility (Fig. $3 \mathrm{~d}$ and Additional file 1: Figure S5a). For multi-gene regulatory regions, there is a competitive regulation of TFs $[50,51]$. When TF is missing, other proteins occupy the vacated sites, thereby maintaining chromatin accessibility. In this case, the transcription of downstream gene is jointly determined by the lack of TF and the newly occupied TFs.

Comparative genomics focuses on the comparison of DNA coding regions between different species, revealing 
the eukaryotic genomic evolution of different Aspergillus species [9]. In comparing open chromatin regions (noncoding sequences) between $A$. niger and A. oryzae species, we discovered that syntenic orthologues of 1564 accessible regions across $A$. niger and $A$. oryzae drive the expression of a collection of target genes that are significantly enriched in housekeeping genes functioning in ribosomes, translation, and energy. The synteny of open chromatin regions between different species may be due to the conservation of noncoding regions [9], indicating that Aspergillus species, which act as protein expression cell factories, exhibit the same potential functional elements in protein synthesis regulation.

The accessible chromatin regions identified by ATACseq are particularly valuable for the identification of cisregulatory DNA elements [32, 48, 49]. By comparing the Tn5 digestion patterns of WT, TF-deleted strains, and naked genome, the strand-specific Tn5 integration patterns around TFBs were only observed in wild-type strain (Fig. 6a). In fact, TF does not exist in the naked genomic DNA and the TF-deleted strain, so the DNA footprints should not be enriched. Given the digestion bias of Tn5 transposase, these footprints are regarded as the pseudo footprints and several research proposed correction methods of TF footprinting [26, 42, 52]. However, there is no consensus on the significance of correcting DNase I or $\operatorname{Tn} 5$ transposase sequence bias. $\mathrm{Li}$ et al. [26] developed HINT-ATAC to measure the amount of the strand cleavage bias for distinct fragment sizes (All, Nfr, $1 \mathrm{~N}$, and $+2 \mathrm{~N}$ ) around distinct intervals near the TF binding site, and the results showed intricate strand-specific cleavage patterns relative to TF binding. Therefore, we reasoned that the strand-specific unbalanced pattern of $\mathrm{Tn} 5$ digestion on both sides of the footprints can be considered a true DNA footprint.

We systematically identified genome-wide DNA footprints from candidate accessible regions and performed de novo motif enrichment analysis on the footprints from A. niger CBS513.88 and SH2 (Fig. 5a, c). Clustering of TFs by family and analysis of $P$ values indicated that the A. niger CBS513.88 and SH2 ATAC-seq footprints were enriched for de novo bZIP (motif GCTGAGTCAG $\mathrm{CV}$ ) and basic helix-loop-helix (motif TCACGTGATC) families (Fig. 5c). The 775 genes predicted as DNAbinding TFs are primarily distributed among 37 classes of regulatory proteins, including 17 bZip family TFs and 10 bHLH family TFs in the A. niger genome $[3,5]$.

The bZip family of TFs that have been functionally analyzed in A. niger mainly include hacA (UPR) and $c p c A$ (amino acid starvation). The function of other bZip TFs are associated with amino acid starvation $(j l b A)$; oxidative stress (napA, atfA, and atfB); and sulfur metabolism (cys-3/metR), and they have mainly been characterized in homologous species, such as A. nidulans [53-55], A. fumigatus [14], and A. oryzae [56, 57]. The gene regulatory network of the bZIP family of Neurospora crassa [58] reveals that the bZIP family of TFs exerts consistent regulatory effects in response to environmental stresses, such as oxidative stress, amino acid starvation, and heavy metal pressure. Functional enrichment of the most proximal gene targeted by the bZip motif, GCTGAGTCAG $\mathrm{CV}$, further demonstrates that this type of TF is associated with transcriptional regulation, which is the first step in regulating the stress response of filamentous fungi to the external environment.

The 10 TFs in the bHLH family, including homologous genes of $s c l R / s r b B$, anbH1, srbA, palcA, INO4, and $\operatorname{dev} R$, have not been well characterized in $A$. niger. However, in other species, the bHLH TFs have been postulated to be involved in hyphae growth and differentiation as well as specific metabolic pathways [36, 59]. The GO category of our E-box targeted genes found that they are involved in the biological process of translation, which is identical to the function of the bHLH family proteins that was predicted following $A$. nidulans genome sequence analysis [9]. Thus, we reasoned that bHLH TFs in $A$. niger have more unknown functions, and further characterization of bHLH TFs is urgently needed. The bHLH TFs recognize a consensus core element named the E-box (5'-CAVVTG-3'), and the Gbox (5'-CACGTG-3') is the most common form [59]; further, specific recognition of the sequence relies on flanking sequences $[60,61]$. The E-box flanking sequences predicted herein contain not only the $5^{\prime} \mathrm{T}$ residue but also the $3^{\prime}$ ATC residues. The $5^{\prime} \mathrm{T}$ residue flanking the G-box determines major specificity by preventing the binding of other bHLH TFs, such as yeast PHO4 [61], whereas the significance of the 3 ' residues is not fully known. The new motif CTGCCTGAGGCA is also a highly enriched motif according to the $P$ value compared to all de novo previously unidentified TF binding motifs. GO functional analysis showed that this new motif CTGCCTGAGGCA drives the expression of target genes, and the GO molecular function categories include the cellular components of "ribosome" and "mitochondrial inner membrane;" the biological processes are related to "translation" and "metabolism."

We constructed a minimal synthetic promoter suitable for filamentous fungi that was based on the yeast minimal promoter [62]. We used this promoter to validate the footprint library-driven UAS elements with TF binding site motifs, which provided a new method for in vivo validation of $A$. niger ATAC-seqpredicted binding sites containing TFBs in the absence of other omics data (DNase-seq, ChIP-seq). Our de novo motifs identified from enriched from ATAC-seq footprints were able to drive the expression of the targeted gene in vivo. 
We further determined the enrichment of known TF binding motifs from ATAC-seq footprints by comparing ATAC-seq data from the A. niger wild-type strain and its TF-deleted strains. Footprints for these known TF binding motifs identified by ATAC-seq data were also verified as functional TFBs in vivo. Compared with $A$. niger CBS513.88, the enrichment $P$ value of the TF motif in A. niger SH2 was significantly higher; the most differential values were observed for the CCAAT box, which represents a gene transcriptional activation domain [23], and the translation-related bHLH family [9]. This suggests that $A$. niger $\mathrm{SH} 2$ is a more suitable host for exogenous protein expression [4] at the TF level. The TF motif enrichment observed after growth in different carbon source conditions and in comparison to TF-deleted strains offer valuable information about TF dynamic responses in addition to the external environment and/or intrinsic regulatory factors. Transcriptome analysis of the known TFs creA, amyR, pacC, and prtT revealed that these transcription factors are involved in decentralized functions and do not cluster in categories directly related to TF function; this is even true for the pathwayspecific transcription factor PrtT, which has a variety of functions that are not directly related to TF function that is clustered. Such results are ubiquitous in other studies using RNA-seq to study the function of transcription factors $[13,50,63]$, which makes it particularly difficult to confirm the specific regulatory functions of transcription factors; as a result, the targeted GO term of a TF can only be determined by combining phenotypic changes [50] or ChIP-seq analysis [12, 13]. These functional clustering results are likely to be the effects of cascades of amplified changes caused by TF defects but are not the direct result of TF/DNA interaction. Through the co-regulation analysis of ATAC-seq and RNA-seq of SH2 TF-deleted strains (Fig. 7a), we effectively identified gene sets related to the corresponding TFs, and using GO clustering analysis, we screened genes and revealed that the obtained clustering results can accurately reflect the function of the experimentally verified $\mathrm{TF}$ (Fig. 7a) [35, 47, 64, 65]. Interestingly, we still predicted a different number of corresponding TF binding motifs in the TF knockout strains (delamyR, delprtT, delcreA, delpacC). This result may be caused by three factors: (i) None of these TFs are pioneers that change chromatin accessibility and the vacancies caused by TF defects are filled by other non-functional proteins. (ii) TF regulation is a complex dynamic process in vivo $[8$, 28], so that even if a TF was knocked out, there may be different TFs with alternate binding at the same DNA site. For example, the AmyR recognizing motif 5'CGGN8(C/A)GG-3' and the PrtT recognizing motif CCG(H)CGG compete for the binding sites in the same target gene during nitrogen and carbon source metabolism regulation [50]. (iii) These binding site identifications may be the result of a false positive. Unexpectedly, we discovered that CreA acts not only as repressors but also as activators, which was confirmed by footprint-driven reporter assays in vivo (Fig. 7c, d). Our results contradict the fact that CreA is the repressor for CCR. It can only be speculated that CreA may play an unknown activation role in gene transcriptional regulation or that the predicted binding sites contain an activated binding motif for other unknown transcription factors.

\section{Conclusions}

These data representing the ATAC-seq landscape of TF footprints will help in the exploration of genome-wide regulation of genes active in filamentous fungi, and the data will help to discover new regulatory factors and their potential sites of action. We demonstrated the usefulness of this resource by testing 25 noncoding DNA sites with predicted TF binding motifs driving reporter gene expression in the host strain. Future experiments will be required to validate the functional activity of more noncoding sites and investigate the role of specific TF footprints in controlling gene expression. The sites with cis-acting elements provide a large number of basic modules for artificially synthesized promoters, and our ATAC-seq data set will provide a rich resource for the identification of functional TFs in A. niger. In the future, ATAC-seq may be effectively applied to study other filamentous fungi whose genomes and transcriptomes are available but whose regulatory mechanisms have not yet been explored.

\section{Methods}

\section{Strains and media}

The specific genotypes of strains used in this study are shown in Additional file 11: Table S10. E. coli Mach 1 T1 (Invitrogen, America) was used for molecular cloning and was cultured in Luria-Bertani (LB) medium (1\% $\mathrm{NaCl}, 1 \%$ tryptone, and $0.5 \%$ yeast extract) with $100 \mu \mathrm{g} /$ $\mathrm{mL}$ ampicillin at $37^{\circ} \mathrm{C}$ (both solid and liquid) while shaking at $200 \mathrm{rpm}$. Conidial Aspergillus niger CBS513.88 and Aspergillus oryzae niaD300 were cultured in PDA medium for spore germination, and they were cultured in DPY (also called YPD) medium (2\% glucose, $1 \%$ peptone, $0.5 \%$ yeast extract, $0.5 \% \mathrm{KH}_{2} \mathrm{PO}_{4}$ and $\left.0.05 \% \mathrm{MgSO}_{4} \cdot 7 \mathrm{H}_{2} \mathrm{O}\right)$ for protoplast preparation. Aconidial Aspergillus niger SH2 $(\Delta k u \Delta p y r G)$ was used as the host for the knockout of transcription factors and was cultured in modified liquid Czapek-Dox medium (CD) containing the following substances to support mycelial growth: $2 \%$ glucose, $0.3 \% \mathrm{NaNO}_{3}, 0.1 \% \mathrm{KH}_{2} \mathrm{PO}_{4}$, $0.05 \% \mathrm{MgSO}_{4} \cdot 7 \mathrm{H}_{2} \mathrm{O}, 0.2 \% \mathrm{KCl}$, and $0.01 \% \mathrm{FeSO}_{4} \cdot 7 \mathrm{H}_{2} \mathrm{O}$. When the OD reached 2.0, $1 \mathrm{ml}$ of mycelia was 
inoculated into $100 \mathrm{ml}$ of DPY medium and grown in a thermostatic shaker at $250 \mathrm{rpm}$ and $30^{\circ} \mathrm{C}$ for $40 \mathrm{~h}$. Mycelia were harvested for protoplast preparation. To study the influence of carbon sources on the chromatin accessibility, the glucose in DPY was replaced with $2 \%$ maltose (MPY) [66] or strains were cultured in $2 \%$ fructose for $2 \mathrm{~h}$, then transferred to $\mathrm{CD}$ with $0.1 \%$ fructose or $\mathrm{CD}$ with $1 \%$ glucose for CCR analysis [67]. A. niger HL-1 [68] was used as the expression host to produce the enzyme product of the glucose oxidase gene goxC (An01g14740). Solid CD (2\% agarose) with 1\% Odianisidine (dissolved in methanol), 18\% glucose, and 90 $\mathrm{U} / \mathrm{mL}$ horseradish peroxidase was used to assess the color reaction following goxC expression.

\section{ATAC-seq library preparation}

The A. niger strain CBS513.88, SH2 (including TF deletion strains and different culture conditions) and $A$. oryzae niaD300 (including laeA deletion and overexpression mutants) were grown to mid-log phase in DPY medium or conditional induction medium. Then, $1 \mathrm{~g}$ of mycelia per library was harvested using miracloth, and they were then rinsed with cold water and $0.8 \mathrm{M} \mathrm{NaCl}$. Protoplasts were prepared by incubating mycelial samples in enzymatic lysis buffer composed of $0.8 \mathrm{M} \mathrm{NaCl}, 2 \%$ cellulase, $1 \%$ helicase, $1 \%$ lyticase, and $0.5 \%$ lysozyme, which were dissolved in the corresponding medium and were then filtered with miracloth and washed with cold STC buffer $(10 \mathrm{mM}$ Tri- $\mathrm{HCl} \mathrm{pH}=7.5$, $1.2 \mathrm{M}$ sorbitol, $50 \mathrm{mM} \mathrm{CaCl}_{2}$ ). Subsequently, $5 \times 10^{4}$ protoplasts (cells) were incubated with $200 \mu \mathrm{l}$ of lysis buffer $(10 \mathrm{mM}$ Tris- $\mathrm{HCl}, \mathrm{pH} 7.4,10 \mathrm{mM} \mathrm{NaCl}, 3 \mathrm{mM}$ $\mathrm{MgCl}_{2}, 0.05 \%$ (v/v) IGEPAL CA-630, $1 \mathrm{mM} \mathrm{PMSF}$ and $1 \times$ PIC (EDTA free)) for $10 \mathrm{~min}$ at $4{ }^{\circ} \mathrm{C}$, washed with lysis buffer (without IGEPAL CA-630), and then incubated with $5 \mu$ l of Tn5 Transposase of TTE Mix V50 in $45 \mu \mathrm{L}$ of $1 \times$ TTBL buffer (TruePrep ${ }^{\circ}$ DNA Library Prep kit V2 for Illumina ${ }^{\circ}$, Vazyme, China) at $37^{\circ} \mathrm{C}$ for $30 \mathrm{~min}$. PCR was subsequently performed as previously described [25]. Libraries were purified with AMPure beads (Agencourt) to remove contaminating primer dimers. All libraries were sequenced with $50 \mathrm{bp}$ paired-end reads on an Illumina Hiseq 2500 platform. For the control $(A$. niger CBS513.88 naked genome DNA), $5 \mathrm{ng}$ of genomic DNA was used for the Tn 5 transposase reaction. All the ATAC-seq data were submitted to the NCBI (Accession number, PRJNA566304, PRJNA587805 and PRJNA692847)

\section{Construction of transcription factor knockout and overexpression strains in Aspergillus}

Different TFs, creA [47], amyR [65], cpcA [69], pacC [64], prtT [35], and laeA [37, 38], were searched on the Aspergillus genome database (www.aspgd.com), and homologous arms for gene knockout were designed based on the 1000-bp sequence flanking the CDS region of each gene, which were then amplified with primer sets listed in Additional file 12: Table S11. The uridine auxotrophic marker pyrG from $A$. nidulans was used as a screening marker for TF knockout. The upstream and downstream homologous arms of each TF obtained by PCR amplification were introduced along with the $p y r G$ marker into a pMD20 T-Vector (TaKaRa, Japan) by NEBuilder (New England Biolabs, America), to obtain TF knockout vectors (pdelcreA, pdelamyR, pdelcpcA, pdelpacC, pdelprtT, and pdellaeA). The knockout cassettes that were linearized with XbaI, EcoRI, ApaI, EcoRI, $E c o R I$, and BamHI were introduced into A. niger $\mathrm{SH} 2$ and $A$. oryzae niaD300 by PEG-mediated protoplast transformation. In brief, protoplasts were prepared as the description in ATAC-seq library preparation. Protoplasts were resuspended in a certain amount of STC, and transformation system was made up of $100 \mu \mathrm{L}$ linearized plasmid, $160 \mu \mathrm{L}$ protoplasts, and $60 \mu \mathrm{L}$ PEG. The mixture was incubated on the ice for $30 \mathrm{~min}$, and then added another $1.5 \mathrm{~mL}$ PEG for $25 \mathrm{~min}$ at the room temperature. Finally, the mixture was pooled down on the hyperosmotic sucrose $\mathrm{CD}$ plate. After 5 days cultivated at $30^{\circ} \mathrm{C}$, transformants were obtained and identified. The cassettes for laeA overexpression digested with ApaI were transformed into A. oryzae niaD300 to yield the OElaeA strain. The TF knockout mutants in which homologous integration occurred were analyzed by PCR amplification for upstream and downstream localization, and they were further validated by qRT-PCR (Additional file 1: Figure S11). The primers used in this study are listed in Additional file 12: Table S11.

\section{Chromatin immunoprecipitation and ChIP-seq library preparations}

To confirm the ATAC-seq data, we constructed PrtTFLAG (CBS513.88 $\Delta$ kusA; prtT-3 × FLAG:pyrG) strain, in which the TF PrtT was drove by PglaA and labelled by $3 \times$ FLAG tagging, for ChIP-seq analysis. Fungal conidia $\left(10^{6} / \mathrm{mL}\right)$ were incubated in $100 \mathrm{~mL}$ DPY media at $30^{\circ} \mathrm{C}$ under shaking at $200 \mathrm{rpm}$ for $40 \mathrm{~h}$. The mycelia was harvested, dried with two layers of miracloth, and transferred into buffer A for cross-linking. Cross-linking was done according to the protocol of [12]. Then samples were frozen in liquid nitrogen and lysed with Bullet Blender with the setting $12,5 \times 3$ min with a 1 -min ice interval in between. The lysed supernatant was collected for sonication (UCD 200 for $30 \mathrm{~min}$ ) in ice water slurry with the program: $10 \mathrm{~S}$ on, $15 \mathrm{~S}$ off. Specific antibody anti-FLAG (Sigma, Anti-FLAG M2) was used for binding with the DNA/PrtT complex. For each standard IP, $50 \mu \mathrm{L}$ chromatin, $2 \mu \mathrm{g}$ antibody, and $450 \mu \mathrm{L}$ lysis buffer were added and were kept on a magnetic stand. 
Precipitated sample was then washed and the immunoprecipitated DNA was digested with RNase A and purified with DNA extract kit (Qiagen, Cat. No. 27104). The extracted DNA was prepared using Illumina multiplex system and sequenced by Illumina HiSeq 2500 (Life Technologies).

\section{RNA extraction, qRT-PCR, and RNA-seq analysis}

A small portion of the mycelium used to prepare the ATAC-seq library was left for RNA extraction. Total RNA was isolated using a HiPure Fungal RNA Kit (Magen, China). Reverse transcription was performed with a PrimeScript RT-PCR Kit (TaKaRa, Japan). Quantitative real-time PCR (qRT-PCR) was performed using an ABI 7500 Fast Real-Time PCR System to analyze TFs and the report gene goxC; the primers used are listed in Additional file 12: Table S11. The resulting libraries were sequenced at the Beijing Genomics Institute (BGI) with $50 \mathrm{bp}$ single-end reads on the BGISEQ-500 (NCBI accession number, PRJNA588127). The RNA quality was assessed with an Agilent Bioanalyzer 2100 system to confirm that all samples had an RNA integrity value (above 7.0). All samples were prepared in duplicate. To obtain FASTQ format clean reads, all generated raw sequencing reads were filtered to remove reads with adaptors, reads in which unknown bases were greater than $10 \%$, and low-quality reads. After filtering, clean reads were mapped to the genome and cDNA using HISAT [70] and Bowtie2 [71]. The gene expression level was quantified by RSEM [72], and the FPKM value of all samples is provided in Additional file 13: Supplementary Table S12. The DESeq2 method [73] was used to screen differentially expressed genes between the two groups.

\section{Mapping and normalization of ATAC-seq data}

After removing adaptors using Trimmomatic [74], $50 \mathrm{bp}$ paired-end ATAC-seq reads were mapped to the Aspergillus genome (Ensembl/ASM285 for A. niger and Ensemble/ASM18445 for $A$. oryzae) using Bowtie2 with parameters "--trim 5 --trim 315 " and other default parameters. All reads aligned to the + strand and the strand were offset by $\pm 5 \mathrm{bp}$, respectively, due to the $\operatorname{Tn} 5$ transposase introducing two cuts that were separated by 9 bp [75]. Mapped reads of SAM output were converted to a BAM format and sorted by Samtools [76]. Duplicate reads were removed using the default parameters of the Picard tools MarkDuplicates program (http:// broadinstitute.github.io/picard/).

To visualize mapped reads, BAM format files were converted to bigwig format using the bamCoverage tool in deepTools2 with a bin size of $1 \mathrm{bp}$ and with RPKM normalization. Heatmaps and average plots from the ATAC-seq data matrix were also generated using the "computeMatrix," "plotHeatmap," and "plotProfile" functions in the deepTools2 package [77]. Genome browser images were made using the integrative Genomics Viewer (IGV) [78] and bigwig files that were processed as described above. Fragments length between the pair-end adaptors were calculated with the program: Samtools view ATAC_sorted.bam | awk '\$9>0' | cut -f 9 | sort | uniq -c | sort -b -k2, 2n $\mid$ sed -e 's/^[|t $]^{*} / /\left.\right|^{\prime}>$ fragment_length_count.txt and the desired length was extracted from SAM files by Python.

\section{Peak calling identified accessible chromatin regions}

Accessible regions and peaks of each sample were identified using MACS2 [79] with the following parameters: -f BAMPE --nomodel --shift 100 --extsize 200. The narrowPeak files from the MACS2 output were used for further analysis. The annotatePeaks.pl tool of the HOMER program [45] was used with the default parameters to annotate the location of the identified peaks overlapping with genomic features, the TSS (transcription start site), the TTS (transcription termination site), exons, introns, and intergenic regions, all of which were annotated in the Ensemble Aspergillus niger CBS513.88 genome. Peaks that were $1 \mathrm{~kb}$ upstream of the TSS were associated with the nearest genes. These genes were then analyzed for overrepresented gene ontology and KEGG pathway using FungiFun 2.2.8 BETA (https://sbi.hkijena.de/fungifun/) [80].

\section{Analysis of differential chromatin accessibility}

To identify differentially mapped reads, we used Diffbind [81]. We used the processed ATAC-seq alignment bam file from Bowtie2 and the narrowPeak file from MACS2 for each sample. MA plots (log2-fold change vs. mean average) were used to visualize changes in chromatin accessibility for all peaks.

\section{Identification of footprints}

Footprints were identified with pyDNase [44] using the default parameters. The Wellington footprinting algorithms [44] in pyDNase searched for TF footprints in areas of the genome that MACS2 identified as peaks. Wellington applied a beta-binomial distribution to estimate footprints and elevated the strand-specific activity of DNase or Tn 5 transposase around transcription factor footprints among accessible chromatin regions.

\section{Design and synthesis of a minimal Aspergillus promoter for functional verification of CREs in vivo}

With reference to the yeast minimal promoter screening system [62], we designed a minimal Aspergillus promoter for functional verification of CREs in vivo (Additional file 1: Figure S9a). The $67 \mathrm{bp} \mathrm{5'}$ UTR of the Aspergillus nidulans TPI gene [82] was used as a TSS. The TATA box and the TSS are separated by a 29-bp distance from 
the TSS in the $a m y B$ (An05g02100) promoter of $A$. niger, which facilitates successful loading of the transcription initiation complex, thereby initiating transcription by RNA polymerase. To ensure that the TATA box can be successfully opened during the transcription process, we manually added $8 \mathrm{AT}$-enriched sequences in front of the TA box to reduce the bond energy of the DNA [62]. These elements were linked together by fusion PCR amplification to form a core promoter region (Additional file 1: Figure S9a). The glucose oxidase goxC (An01g14740) was used as the reporter gene, which could catalyze the oxidation of D-glucose at carbon 1 into D-glucono-1, 5-lactone and hydrogen peroxide. Then the peroxidase catalyzes the decomposition of hydrogen peroxide while oxidizing O-anisidine, thus resulting in a red phenotype [83] (Additional file 1: Figure $\mathrm{S} 9 \mathrm{~b})$. To further refine the entire reporting system, the selection marker pyrG was integrated before the footprints to separate the footprints from other genomic sequences, ensuring that transcription of the core sequence was not affected by other sequences. The footprints contained a transcription factor binding site (TFBS) that helped stabilize RNAP to enhance transcription efficiency. By constantly changing footprints and monitoring the transcription of downstream reporter genes, it was possible to identify whether the footprints containing the TFBs function in vivo. The $1 \mathrm{~kb}$ flanking sequence of the glucoamylase gene (glaA ) acts as a homologous arm, and the aim was that the sequence successfully integrated into the glucoamylase gene locus, ensuring that all open reading frames were at the same position in the genome [84]. All the report plasmids were linearized with $\mathrm{XbaI}$ and transformed into $A$. niger by PEG-mediated protoplast transformation.

\section{Discovery of de novo transcription factor motifs}

ATAC-seq footprints that were identified from each sample were used to discover de novo transcription factor motifs. The boundaries of the start and stop coordinates of ATAC-seq footprints were lengthened by $10 \mathrm{bp}$ upstream and downstream. We then used the findMotifsGenome.pl tool of the HOMER program to identify overrepresented transcription factor sites (transcription factor motifs). Using the annotatePeaks.pl tool of HOMER with the -m parameter, HOMER de novo motifs were then used to search the original set of sequences to identify all instances of motifs.

\section{Analysis of known motif enrichment within footprints}

The known motif files in the HOMER format were created manually with the seq2profile.pl tool of HOMER. The known motifs were then used to search the footprints of each sample to identify all instances of motifs using the annotatePeaks.pl tool of HOMER with the $-\mathrm{m}$ parameter.

\section{Abbreviations}

ATAC-seq: Assay for transposase-accessible chromatin using sequencing; TF: Transcription factor; TFBs: Transcription factor binding sites; ChIPseq: Chromatin immunoprecipitation sequencing; CREs: cis-regulatory elements; TSS: Transcription start site; TTS: Transcription terminator site; SMs: Secondary metabolites; PKSs: Polyketide synthases; NRPSs: Nonribosomal peptide synthetases; AF: Aflatoxin; DEGs: Differential expression genes; OC: Open chromatin; CCR: Carbon catabolite repression

\section{Supplementary Information}

The online version contains supplementary material available at https://doi. org/10.1186/s12915-021-01114-0.

\begin{abstract}
Additional file 1: Figure S1. Fragment length distribution of Tn5 transposase integration. Figure S2. qRT-PCR analysis of $A$. niger cultured in different condition. Figure S3. Enrichment of PrtT binding motif from the ChIP-seq data. Figure S4. Visually display of the PrtT binding site. Figure S5. Clustering analysis of ATAC-seq peaks for Aspergillus oryzae niaD300 and its laeA mutants. Figure S6. Comparison of ATAC-seq peaks and RNA-seq signal among A.oryzae niaD300 and laeA mutant. Figure S7. De novo motifs of SH2 TF-deficient strains. Figure S8. GO analysis of 3 de novo predicted over-represented TFBs targeting genes. Figure S9. The minimal promoter structure and the verification of its function. Figure S10. In vivo functional verification of AreA targeting sites. Figure S11. qRT-PCR verification of TF knockout strains of A.niger SH2 and A.oryzae niaD300
\end{abstract}

Additional file 2: Supplementary Table S1. Sequencing data quality and mapping rate analysis of the ATAC-seq samples.

Additional file 3: Supplementary Table S2. Chromatin accessible regions (Peaks) of each sample identified by MACS2.

Additional file 4: Supplementary Table S3. Syntenic orthologues of ATAC-seq peaks identified between A. niger CBS513.88 and A. oryzae niaD300.

Additional file 5: Supplementary Table S4. Differential peaks analysis of $A$. niger and its mutants.

Additional file 6: Supplementary Table S5. Differential peaks and SM cluster analysis of $A$. oryzae and its laeA mutants.

Additional file 7: Supplementary Table S6. DNA footprints of $A$. niger wild-type strains and TF-deleted strains.

Additional file 8: Supplementary Table S7. De novo TFBs of A. niger wild-type strains and TF-deleted strains with a $P$ value threshold of $1 E-50$ by HOMER.

Additional file 9: Supplementary Table S8. The physical position and gene function of DNA instances containing the TFBs mapped to the $A$. niger genome.

Additional file 10: Supplementary Table S9. Motif instances of known TFs from footprints by comparing A. niger WT strains and the TFdeleted strains.

Additional file 11: Supplementary Table S10. Genotype of strains used in this study.

Additional file 12: Supplementary Table S11. Primers used in this study

Additional file 13: Supplementary Table S12. FPKM value of all the A. niger RNA-seq samples.

Additional file 14: Supplementary Table S13. Original data of all the figures and additional files.

Acknowledgements

We are very grateful to Vazyme Biotech Co., Ltd (Nanjing, China) for providing the Tn5 reaction kit and the corresponding technicians. We also thank BGI Company for NGS services. 


\section{Authors' contributions}

LH was responsible for conception and design, acquisition of the data, analysis and interpretation of the data, and drafting of the article. $X L, L Y$, and LD were responsible for acquisition of the data, analysis, and interpretation of the data. BW and LP responsible for conception and design, acquisition of the data, analysis and interpretation of the data, and drafting of the article. All authors read and approved the final manuscript.

\section{Funding}

This work was supported by the National Natural Science Foundation of China (grant number 31871736, 31870024], the Science and Technology Planning Project of Tianjin City (grant no. TSBICIP-KJGG-006-21), the Core Technology Project of Foshan City (project no.1920001000824), and the Science and Technology Planning Project of Guangzhou City (grant number 202002030207).

\section{Availability of data and materials}

All data generated or analyzed during this study are included in this published article, its supplementary information files, and publicly available repositories. The raw ATAC-seq and ChIP-seq sequencing data have been deposited in the NCBI SRA database under the accession number PRJNA566304, PRJNA587805, PRJNA692847, and PRJNA692789. The raw RNAseq sequencing data have been deposited in the NCBI SRA database under the accession number PRJNA588127. The Supporting data are included in the additional files $2,3,4,5,6,7,8,9,10,11,12$ and 13 . A file providing individual data values was submitted as Additional file 14 .

\section{Declarations}

Ethics approval and consent to participate

Not applicable.

\section{Consent for publication}

Not applicable.

\section{Competing interests}

The authors declare that no competing interests exist.

\section{Author details}

${ }^{1}$ School of Biology and Biological Engineering, South China University of Technology, Guangzhou Higher Education Mega Center, Guangzhou 510006, China. ${ }^{2}$ Guangdong Provincial Key Laboratory of Fermentation and Enzyme Engineering, South China University of Technology, Guangzhou Higher Education Mega Center, Guangzhou 510006, China.

Received: 8 January 2020 Accepted: 2 August 2021

Published online: 06 September 2021

\section{References}

1. Andersen MR, Salazar MP, Schaap PJ, van de Vondervoort PJ, Culley D, Thykaer J, et al. Comparative genomics of citric-acid-producing Aspergillus niger ATCC 1015 versus enzyme-producing CBS 513.88. Genome Res. 2011; 21(6):885-97. https://doi.org/10.1101/gr.112169.110.

2. Vesth TC, Nybo JL, Theobald S, Frisvad JC, Larsen TO, Nielsen KF, et al. Investigation of inter- and intraspecies variation through genome sequencing of Aspergillus section Nigri. Nat Genet. 2018;50(12):1688-95. https://doi.org/10.1038/s41588-018-0246-1.

3. Pel HJ, de Winde JH, Archer DB, Dyer PS, Hofmann G, Schaap PJ, et al. Genome sequencing and analysis of the versatile cell factory Aspergillus niger CBS 513.88. Nat Biotechnol. 2007;25(2):221-31. https://doi.org/10.1038/ nbt1282.

4. Yin C, Wang B, He P, Lin Y, Pan L. Genomic analysis of the aconidial and highperformance protein producer, industrially relevant Aspergillus niger SH2 strain. Gene. 2014;541(2):107-14. https://doi.org/10.1016/j.gene.2014.03.011.

5. Todd RB, Zhou M, Ohm RA, Leeggangers HA, Visser L, de Vries RP. Prevalence of transcription factors in ascomycete and basidiomycete fungi. BMC Genomics. 2014;15(1):214. https://doi.org/10.1186/1471-2164-15-214.

6. Schape P, Kwon MJ, Baumann B, Gutschmann B, Jung S, Lenz S, et al. Updating genome annotation for the microbial cell factory Aspergillus niger using gene co-expression networks. Nucleic Acids Res. 2019;47(2):559-69. https://doi.org/10.1093/nar/gky1183.
7. Felsenfeld G. Chromatin as an essential part of the transcriptional mechanism. Nature. 1992;355(6357):219-24. https://doi.org/10.1038/355219a 0 .

8. Qiu Z, Li R, Zhang S, Wang K, Xu M, Li J, et al. Identification of regulatory DNA elements using genome-wide mapping of DNase I hypersensitive sites during tomato fruit development. Mol Plant. 2016;9(8):1168-82. https://doi. org/10.1016/j.molp.2016.05.013.

9. Galagan JE, Calvo SE, Cuomo C, Ma LJ, Wortman JR, Batzoglou S, et al. Sequencing of Aspergillus nidulans and comparative analysis with $A$. fumigatus and A. oryzae. Nature. 2005;438(7071):1105-15. https://doi.org/10.1 038/nature04341.

10. Johnson DS, Mortazavi A, Myers RM, Wold B. Genome-wide mapping of in vivo protein-DNA interactions. Science. 2007;316(5830):1497-502. https:// doi.org/10.1126/science.1141319.

11. Park PJ. ChIP-seq: advantages and challenges of a maturing technology. Nat Rev Genet. 2009;10(10):669-80. https://doi.org/10.1038/nrg2641.

12. de Castro PA, Chen C, de Almeida RS, Freitas FZ, Bertolini MC, Morais ER, et al. ChIP-seq reveals a role for CrzA in the Aspergillus fumigatus highosmolarity glycerol response (HOG) signalling pathway. Mol Microbiol. 2014; 94(3):655-74. https://doi.org/10.1111/mmi.12785.

13. Chung D, Barker BM, Carey CC, Merriman B, Werner ER, Lechner BE, et al. ChIP-seq and in vivo transcriptome analyses of the Aspergillus fumigatus SREBP SrbA reveals a new regulator of the fungal hypoxia response and virulence. PLoS Pathog. 2014;10(11):e1004487. https://doi.org/10.1371/journa I.ppat.1004487.

14. Amich J, Schafferer L, Haas H, Krappmann S. Regulation of sulphur assimilation is essential for virulence and affects iron homeostasis of the human-pathogenic mould Aspergillus fumigatus. PLoS Pathog. 2013;9(8): e1003573. https://doi.org/10.1371/journal.ppat.1003573.

15. Paul S, Stamnes M, Thomas GH, Liu H, Hagiwara D, Gomi K, et al. AtrR is an essential determinant of azole resistance in Aspergillus fumigatus. mBio. 2019;10(2). https://doi.org/10.1128/mbio.02563-18.

16. Wu MY, Mead ME, Lee MK, Ostrem Loss EM, Kim SC, Rokas A, et al. Systematic dissection of the evolutionarily conserved WetA developmental regulator across a genus of filamentous fungi. mBio. 2018;9(4). https://doi. org/10.1128/mBio.01130-18.

17. Sun J, Glass NL. Identification of the CRE-1 cellulolytic regulon in Neurospora crassa. PLoS One. 2011;6(9):e25654. https://doi.org/10.1371/journal.pone.002 5654 .

18. Nasmith CG, Walkowiak S, Wang L, Leung WW, Gong Y, Johnston A, et al. Tri6 is a global transcription regulator in the phytopathogen Fusarium graminearum. PLoS Pathog. 2011;7(9):e1002266. https://doi.org/10.1371/ journal.ppat.1002266.

19. Kim S, Hu J, Oh Y, Park J, Choi J, Lee YH, et al. Combining ChIP-chip and expression profiling to model the MoCRZ1 mediated circuit for $\mathrm{Ca} /$ calcineurin signaling in the rice blast fungus. PLoS Pathog. 2010;6(5): e1000909. https://doi.org/10.1371/journal.ppat.1000909.

20. Gacek-Matthews A, Berger H, Sasaki T, Wittstein K, Gruber C, Lewis ZA, et al. $\mathrm{KdmB}$, a jumonji histone $\mathrm{H} 3$ demethylase, regulates genome-wide $\mathrm{H} 3 \mathrm{~K} 4$ trimethylation and is required for normal induction of secondary metabolism in Aspergillus nidulans. PLoS Genet. 2016;12(8):e1006222. https:// doi.org/10.1371/journal.pgen.1006222.

21. Fischer J, Muller SY, Netzker T, Jager N, Gacek-Matthews A, Scherlach K, et al. Chromatin mapping identifies BasR, a key regulator of bacteriatriggered production of fungal secondary metabolites. eLife. 2018;7:e40969. https://doi.org/10.7554/eLife.40969.

22. Giresi PG, Kim J, McDaniell RM, lyer VR, Lieb JD. FAIRE (formaldehydeassisted isolation of regulatory elements) isolates active regulatory elements from human chromatin. Genome Res. 2007;17(6):877-85. https://doi.org/1 $0.1101 /$ gr.5533506.

23. Wang C, Lv Y, Wang B, Yin C, Lin Y, Pan L. Survey of protein-DNA interactions in Aspergillus oryzae on a genomic scale. Nucleic Acids Res. 2015;43(9):4429-46. https://doi.org/10.1093/nar/gkv334.

24. Zentner GE, Henikoff S. High-resolution digital profiling of the epigenome Nat Rev Genet. 2014;15(12):814-27. https://doi.org/10.1038/nrg3798.

25. Buenrostro JD, Wu B, Chang HY, Greenleaf WJ. ATAC-seq: A method for assaying chromatin accessibility genome-wide. Curr Protocols Mol Biol. 2015;109:21 9 1-9.

26. Li Z, Schulz MH, Look T, Begemann M, Zenke M, Costa IG. Identification of transcription factor binding sites using ATAC-seq. Genome Biol. 2019;20(1): 45. https://doi.org/10.1186/s13059-019-1642-2. 
27. Buenrostro JD, Giresi PG, Zaba LC, Chang HY, Greenleaf WJ. Transposition of native chromatin for fast and sensitive epigenomic profiling of open chromatin, DNA-binding proteins and nucleosome position. Nat Methods. 2013;10(12):1213-8. https://doi.org/10.1038/nmeth.2688.

28. Wu J, Huang B, Chen H, Yin Q, Liu Y, Xiang Y, et al. The landscape of accessible chromatin in mammalian preimplantation embryos. Nature. 2016; 534(7609):652-7. https://doi.org/10.1038/nature18606.

29. Fernandez-Minan A, Bessa J, Tena JJ, Gomez-Skarmeta JL. Assay for transposase-accessible chromatin and circularized chromosome conformation capture, two methods to explore the regulatory landscapes of genes in zebrafish. Methods Cell Biol. 2016;135:413-30. https://doi.org/1 0.1016/bs.mcb.2016.02.008.

30. Bozek M, Cortini R, Storti AE, Unnerstall U, Gaul U, Gompel N. ATAC-seq reveals regional differences in enhancer accessibility during the establishment of spatial coordinates in the Drosophila blastoderm. Genome Res. 2019;29(5):771-83. https://doi.org/10.1101/gr.242362.118.

31. Daugherty AC, Yeo RW, Buenrostro JD, Greenleaf WJ, Kundaje A, Brunet A. Chromatin accessibility dynamics reveal novel functional enhancers in $C$. elegans. Genome Res. 2017;27(12):2096-107. https://doi.org/10.1101/gr.22 6233.117 .

32. Tannenbaum M, Sarusi-Portuguez A, Krispil R, Schwartz M, Loza O, Benichou $\mathrm{JIC}$, et al. Regulatory chromatin landscape in Arabidopsis thaliana roots uncovered by coupling INTACT and ATAC-seq. Plant Methods. 2018;14(1): 113. https://doi.org/10.1186/s13007-018-0381-9.

33. Schep AN, Buenrostro JD, Denny SK, Schwartz K, Sherlock G, Greenleaf WJ. Structured nucleosome fingerprints enable high-resolution mapping of chromatin architecture within regulatory regions. Genome Res. 2015;25(11): 1757-70. https://doi.org/10.1101/gr.192294.115.

34. Mavrich TN, loshikhes IP, Venters BJ, Jiang C, Tomsho LP, Qi J, et al. A barrier nucleosome model for statistical positioning of nucleosomes throughout the yeast genome. Genome Res. 2008;18(7):1073-83. https://doi.org/10.11 01/gr.078261.108.

35. Caruso ML, Litzka O, Martic G, Lottspeich F, Brakhage AA. Novel basic-region helix-loop-helix transcription factor ( $\mathrm{AnBH} 1$ ) of Aspergillus nidulans counteracts the CCAAT-binding complex AnCF in the promoter of a penicillin biosynthesis gene. J Mol Biol. 2002;323(3):425-39. https://doi.org/1 0.1016/50022-2836(02)00965-8.

36. Bok JW, Keller NP. LaeA, a regulator of secondary metabolism in Aspergillus spp. Eukaryot Cell. 2004;3(2):527-35. https://doi.org/10.1128/EC.3.2.527-535.2 004.

37. Bok JW, Hoffmeister D, Maggio-Hall LA, Murillo R, Glasner JD, Keller NP. Genomic mining for Aspergillus natural products. Chem Biol. 2006;13(1):31-7. https://doi.org/10.1016/j.chembiol.2005.10.008.

38. Wang $B, L V Y$, Li X, Lin Y, Deng H, Pan L. Profiling of secondary metabolite gene clusters regulated by LaeA in Aspergillus niger FGSC A1279 based on genome sequencing and transcriptome analysis. Res Microbiol. 2018;169(2): 67-77. https://doi.org/10.1016/j.resmic.2017.10.002.

39. Patananan AN, Palmer JM, Garvey GS, Keller NP, Clarke SG. A novel automethylation reaction in the Aspergillus nidulans LaeA protein generates S-methylmethionine. J Biol Chem. 2013;288(20):14032-45. https://doi.org/1 0.1074/jbc.M113.465765.

40. Khaldi N, Seifuddin FT, Turner G, Haft D, Nierman WC, Wolfe KH, et al. SMURF: Genomic mapping of fungal secondary metabolite clusters. Fungal Genet Biol. 2010;47(9):736-41. https://doi.org/10.1016/j.fgb.2010. 06.003.

41. Karabacak Calviello A, Hirsekorn A, Wurmus R, Yusuf D, Ohler U. Reproducible inference of transcription factor footprints in ATAC-seq and DNase-seq datasets using protocol-specific bias modeling. Genome Biol. 2019;20(1):42. https://doi.org/10.1186/s13059-019-1654-y.

42. Lu Z, Hofmeister BT, Vollmers C, DuBois RM, Schmitz RJ. Combining ATACseq with nuclei sorting for discovery of cis-regulatory regions in plant genomes. Nucleic Acids Res. 2017;45(6):e41. https://doi.org/10.1093/nar/ gkw1179.

43. Piper J, Elze MC, Cauchy P, Cockerill PN, Bonifer C, Ott S. Wellington: a novel method for the accurate identification of digital genomic footprints from DNase-seq data. Nucleic Acids Res. 2013;41 (21):e201. https://doi.org/10.1 093/nar/gkt850.

44. Heinz S, Benner C, Spann N, Bertolino E, Lin YC, Laslo P, et al. Simple combinations of lineage-determining transcription factors prime cisregulatory elements required for macrophage and B cell identities. Mol Cell. 2010;38(4):576-89. https://doi.org/10.1016/j.molcel.2010.05.004.
45. Zhou B, Wang C, Wang B, Li X, Xiao J, Pan L. Identification of functional ciselements required for repression of the Taka-amylase $\mathrm{A}$ gene under secretion stress in Aspergillus oryzae. Biotechnol Lett. 2015;37(2):333-41. https://doi.org/10.1007/s10529-014-1691-2.

46. Drysdale MR, Kolze SE, Kelly JM. The Aspergillus niger carbon catabolite repressor encoding gene, creA. Gene. 1993;130(2):241-5. https://doi.org/10.1 016/0378-1119(93)90425-3.

47. Ackermann AM, Wang Z, Schug J, Naji A, Kaestner KH. Integration of ATACseq and RNA-seq identifies human alpha cell and beta cell signature genes. Mol Metab. 2016;5(3):233-44. https://doi.org/10.1016/j.molmet.2016.01.002.

48. Toenhake CG, Fraschka SA, Vijayabaskar MS, Westhead DR, van Heeringen SJ, Bartfai R. Chromatin accessibility-based characterization of the gene regulatory network underlying plasmodium falciparum blood-stage development. Cell Host Microbe. 2018;23(4):557-69 e9. https://doi.org/10.1 016/j.chom.2018.03.007.

49. Chen L, Zou G, Zhang L, de Vries RP, Yan X, Zhang J, et al. The distinctive regulatory roles of PrtT in the cell metabolism of Penicillium oxalicum. Fungal Genet Biol. 2014;63:42-54. https://doi.org/10.1016/j.fgb.2013.12.001.

50. Huang L, Dong L, Wang B, Pan L. The transcription factor PrtT and its target protease profiles in Aspergillus niger are negatively regulated by carbon sources. Biotechnol Lett. 2020;42(4):613-24. https://doi.org/10.1007/s10529020-02806-3.

51. Gusmao EG, Allhoff M, Zenke M, Costa IG. Analysis of computational footprinting methods for DNase sequencing experiments. Nat Methods. 2016;13(4):303-9. https://doi.org/10.1038/nmeth.3772.

52. Strittmatter AW, Irniger S, Braus GH. Induction of jlbA mRNA synthesis for a putative bZIP protein of Aspergillus nidulans by amino acid starvation. Curr Genet. 2001;39(5-6):327-34. https://doi.org/10.1007/s002940100215.

53. Balazs A, Pocsi I, Hamari Z, Leiter E, Emri T, Miskei M, et al. AtfA bZIP-type transcription factor regulates oxidative and osmotic stress responses in Aspergillus nidulans. Mol Gen Genomics. 2010;283(3):289-303. https://doi. org/10.1007/s00438-010-0513-z.

54. Mendoza-Martinez AE, Lara-Rojas F, Sanchez O, Aguirre J. NapA mediates a redox regulation of the antioxidant response, carbon utilization and development in Aspergillus nidulans. Front Microbiol. 2017;8:516. https://doi. org/10.3389/fmicb.2017.00516.

55. Sakamoto K, Arima TH, Iwashita K, Yamada O, Gomi K, Akita O. Aspergillus oryzae atfB encodes a transcription factor required for stress tolerance in conidia. Fungal Genet Biol. 2008;45(6):922-32. https://doi.org/10.1016/j.fgb.2 008.03.009.

56. Sakamoto K, Iwashita K, Yamada O, Kobayashi K, Mizuno A, Akita O, et al. Aspergillus oryzae atfA controls conidial germination and stress tolerance. Fungal Genet Biol. 2009;46(12):887-97. https://doi.org/10.1016/j.fgb.2009.09. 004.

57. Tian C, Li J, Glass NL. Exploring the bZIP transcription factor regulatory network in Neurospora crassa. Microbiology. 2011;157(Pt 3):747-59. https:// doi.org/10.1099/mic.0.045468-0.

58. Jin FJ, Takahashi T, Matsushima K, Hara S, Shinohara Y, Maruyama J, et al. ScIR, a basic helix-loop-helix transcription factor, regulates hyphal morphology and promotes sclerotial formation in Aspergillus oryzae. Eukaryot Cell. 2011;10(7):945-55. https://doi.org/10.1128/EC.00013-11.

59. Fisher F, Goding CR. Single amino acid substitutions alter helix-loop-helix protein specificity for bases flanking the core CANNTG motif. EMBO J. 1992; 11(11):4103-9. https://doi.org/10.1002/j.1460-2075.1992.tb05503.x.

60. Zhou X, O'Shea EK. Integrated approaches reveal determinants of genomewide binding and function of the transcription factor Pho4. Mol Cell. 2011; 42(6):826-36. https://doi.org/10.1016/j.molcel.2011.05.025.

61. Redden H, Alper HS. The development and characterization of synthetic minimal yeast promoters. Nat Commun. 2015;6(1):7810. https://doi.org/10.1 038/ncomms8810.

62. Carvalho ND, Jorgensen TR, Arentshorst M, Nitsche BM, van den Hondel CA, Archer DB, et al. Genome-wide expression analysis upon constitutive activation of the HacA bZIP transcription factor in Aspergillus niger reveals a coordinated cellular response to counteract ER stress. BMC Genomics. 2012; 13(1):350. https://doi.org/10.1186/1471-2164-13-350.

63. MacCabe AP, Van den Hombergh JP, Tilburn J, Arst HN Jr, Visser J. Identification, cloning and analysis of the Aspergillus niger gene pacC, a wide domain regulatory gene responsive to ambient $\mathrm{pH}$. Mol Gen Genet. 1996;250(3):367-74. https://doi.org/10.1007/BF02174395.

64. Punt PJ, Schuren FH, Lehmbeck J, Christensen T, Hiort C, van den Hondel CA. Characterization of the Aspergillus niger prtT, a unique regulator of 
extracellular protease encoding genes. Fungal Genet Biol. 2008;45(12):15919. https://doi.org/10.1016/j.fgb.2008.09.007.

65. vanKuyk PA, Benen JA, Wosten HA, Visser J, de Vries RP. A broader role for AmyR in Aspergillus niger: regulation of the utilisation of D-glucose or Dgalactose containing oligo- and polysaccharides. Appl Microbiol Biotechnol. 2012:93(1):285-93. https://doi.org/10.1007/s00253-011-3550-6.

66. Suzuki K, Tanaka M, Konno Y, Ichikawa T, Ichinose S, Hasegawa-Shiro S, et al. Distinct mechanism of activation of two transcription factors, AmyR and MalR, involved in amylolytic enzyme production in Aspergillus oryzae. Appl Microbiol Biotechnol. 2015;99(4):1805-15. https://doi.org/10.1007/s00253014-6264-8.

67. Strauss J, Horvath HK, Abdallah BM, Kindermann J, Mach RL, Kubicek CP. The function of CreA, the carbon catabolite repressor of Aspergillus nidulans, is regulated at the transcriptional and post-transcriptional level. Mol Microbiol. 1999:32(1):169-78. https://doi.org/10.1046/j.1365-2958.1999.01341. $x$.

68. Dong L, Yu D, Lin X, Wang B, Pan L. Improving expression of thermostable trehalase from Myceliophthora sepedonium in Aspergillus niger mediated by the CRISPR/Cas9 tool and its purification, characterization. Protein Expr Purif. 2019;165:105482

69. Wanke C, Eckert S, Albrecht G, van Hartingsveldt W. Punt PJ, van den Hondel CAMJ, et al. The Aspergillus niger GCN4 homologue, $c p c A$, is transcriptionally regulated and encodes an unusual leucine zipper. Mol Microbiol. 1997;23(1):23-33. https://doi.org/10.1046/j.1365-2958.1997.174154 9.x.

70. Kim D, Landmead B, Salzberg SL. HISAT: a fast spliced aligner with low memory requirements. Nat Methods. 2015;12(4):357-U121. https://doi.org/1 0.1038/nmeth.3317.

71. Langmead B, Salzberg SL. Fast gapped-read alignment with Bowtie 2. Nat Methods. 2012;9(4):357-9. https://doi.org/10.1038/nmeth.1923.

72. Li B, Dewey CN. RSEM: accurate transcript quantification from RNA-Seq data with or without a reference genome. BMC Bioinformatics. 2011;12(1):323. https://doi.org/10.1186/1471-2105-12-323.

73. Love MI, Huber W, Anders S. Moderated estimation of fold change and dispersion for RNA-seq data with DESeq2. Genome Biol. 2014;15(12):550. https://doi.org/10.1186/s13059-014-0550-8.

74. Bolger AM, Lohse M, Usadel B. Trimmomatic: a flexible trimmer for Illumina sequence data. Bioinformatics. 2014;30(15):2114-20. https://doi.org/10.1093/ bioinformatics/btu170

75. Adey A, Morrison HG, Asan, Xun X, Kitzman JO, Turner EH, et al. Rapid, lowinput, low-bias construction of shotgun fragment libraries by high-density in vitro transposition. Genome Biol. 2010;11(12):R119. https://doi.org/10.11 86/gb-2010-11-12-r119.

76. Li H, Handsaker B, Wysoker A, Fennell T, Ruan J, Homer N, et al. The sequence alignment/map format and SAMtools. Bioinformatics. 2009;25(16): 2078-9. https://doi.org/10.1093/bioinformatics/btp352.

77. Ramirez F, Ryan DP, Gruning B, Bhardwaj V, Kilpert F, Richter AS, et al. deepTools2: a next generation web server for deep-sequencing data analysis. Nucleic Acids Res. 2016;44(W1):W160-W5. https://doi.org/10.1093/ nar/gkw257.

78. Thorvaldsdottir H, Robinson JT, Mesirov JP. Integrative Genomics Viewer (IGV): high-performance genomics data visualization and exploration. Brief Bioinform. 2013:14(2):178-92. https://doi.org/10.1093/bib/bbs017.

79. Zhang Y, Liu T, Meyer CA, Eeckhoute J, Johnson DS, Bernstein BE, et al. Model-based Analysis of ChIP-Seq (MACS). Genome Biol. 2008;9(9):R137. https://doi.org/10.1186/gb-2008-9-9-r137.

80. Priebe S, Kreisel C, Horn F, Guthke R, Linde J. FungiFun2: a comprehensive online resource for systematic analysis of gene lists from fungal species. Bioinformatics. 2015;31(3):445-6. https://doi.org/10.1093/bioinformatics/ btu627.

81. Ross-Innes CS, Stark R, Teschendorff AE, Holmes KA, Ali HR, Dunning MJ, et al. Differential oestrogen receptor binding is associated with clinical outcome in breast cancer. Nature. 2012;481(7381):389-U177. https://doi. org/10.1038/nature10730

82. McKnight GL, O'Hara PJ, Parker ML. Nucleotide sequence of the triosephosphate isomerase gene from Aspergillus nidulans: implications for a differential loss of introns. Cell. 1986;46(1):143-7. https://doi.org/10.1016/ 0092-8674(86)90868-8.

83. Guo Y, Lu F, Zhao H, Tang Y, Lu Z. Cloning and heterologous expression of glucose oxidase gene from Aspergillus niger Z-25 in Pichia pastoris. Appl
Biochem Biotechnol. 2010;162(2):498-509. https://doi.org/10.1007/s12010009-8778-6.

84. Rantasalo A, Landowski CP, Kuivanen J, Korppoo A, Reuter L, Koivistoinen O, et al. A universal gene expression system for fungi. Nucleic Acids Res. 2018; 46(18):e111. https://doi.org/10.1093/nar/gky558.

\section{Publisher's Note}

Springer Nature remains neutral with regard to jurisdictional claims in published maps and institutional affiliations.
Ready to submit your research? Choose BMC and benefit from:

- fast, convenient online submission

- thorough peer review by experienced researchers in your field

- rapid publication on acceptance

- support for research data, including large and complex data types

- gold Open Access which fosters wider collaboration and increased citations

- maximum visibility for your research: over $100 \mathrm{M}$ website views per year

At $\mathrm{BMC}$, research is always in progress.

Learn more biomedcentral.com/submissions 